\title{
An S-0psin Knock-In Mouse (F81Y) Reveals a Role for the Native Ligand 11-cis-Retinal in Cone Opsin Biosynthesis
}

\author{
Christine Insinna, ${ }^{1 \star}$ Lauren L. Daniele, ${ }^{1 \star}$ Jason A. Davis, ${ }^{2}$ DeLaine D. Larsen, ${ }^{1}$ Colleen Kuemmel, ${ }^{3}$ Jinhua Wang, ${ }^{4}$ \\ Sergei S. Nikonov, ${ }^{5}$ Barry E. Knox, ${ }^{3}$ and Edward N. Pugh Jr. ${ }^{1}$ \\ ${ }^{1}$ Center for Neuroscience, University of California, Davis, California 95618, ${ }^{2}$ Department of Ophthalmology, University of Pennsylvania, Philadelphia, \\ Pennsylvania 19104, ${ }^{3}$ Department of Neuroscience and Physiology, State University of New York Upstate Medical University, Syracuse, New York 13210, \\ and ${ }^{4}$ Department of Pathology and Laboratory Medicine, and ${ }^{5}$ Department of Neuroscience, University of Pennsylvania, Philadelphia, Pennsylvania 19104
}

In absence of their natural ligand, 11-cis-retinal, cone opsin G-protein-coupled receptors fail to traffic normally, a condition associated with photoreceptor degeneration and blindness. We created a mouse with a point mutation (F81Y) in cone S-opsin. As expected, cones with this knock-in mutation respond to light with maximal sensitivity red-shifted from 360 to $420 \mathrm{~nm}$, consistent with an altered interaction between the apoprotein and ligand, 11-cis-retinal. However, cones expressing F81Y S-opsin showed an $\sim 3$-fold reduced absolute sensitivity that was associated with a corresponding reduction in S-opsin protein expression. The reduced S-opsin expression did not arise from decreased S-opsin mRNA or cone degeneration, but rather from enhanced endoplasmic reticulum (ER)-associated degradation of the nascent protein. Exogenously increased 11-cis-retinal restored F81Y S-opsin protein expression to normal levels, suggesting that ligand binding in the ER facilitates proper folding. Immunohistochemistry and electron microscopy of normal retinas showed that Mueller cells, which synthesize a precursor of 11-cis-retinal, are closely adjoined to the cone ER, so they could deliver the ligand to the site of opsin synthesis. Together, these results suggest that the binding of 11-cis-retinal in the ER is important for normal folding during cone opsin biosynthesis.

\section{Introduction}

Opsins are G-protein-coupled receptors (GPCRs) that initiate vision via a signaling cascade whose molecular components are highly concentrated in an elaborated cilium, the photoreceptor outer segment. The synthesis of these components creates a large daily burden of transcription, translation, protein trafficking, and turnover, with total opsin renewed at a rate of about 10\% per day (Young, 1967, 1971; Hollyfield, 1979; Jonnal et al., 2010). More than 100 mutations in rhodopsin have been associated with retinal degeneration, typically autosomal dominant retinitis pigmentosa (Hartong et al., 2006), and most of these mutations can be characterized as causing opsin misfolding (Rakoczy et al., 2011). An increasing number of cone opsin mutations are associated with retinal disease, thanks to the growing application of high-resolution fundus imaging, high-throughput genotyping, and other novel methodologies (Carroll et al., 2009; Gardner et al., 2010). Because

Received Jan. 9, 2012; revised April 6, 2012; accepted April 24, 2012.

Author contributions: C.I., L.L.D., J.A.D., J.W., S.S.N., B.E.K., and E.N.P. designed research; C.I., L.L.D., J.A.D., D.D.L., C.K., J.W., S.S.N., B.E.K., and E.N.P. performed research; C.I, L.L.D., J.A.D., D.D.L., C.K., J.W., S.S.N., B.E.K., and E.N.P. analyzed data; C.I., L.L.D., J.A.D., and E.N.P. wrote the paper.

This work was supported by National Institutes of Health Grant EY02660. We are grateful to Marie Burns for helpful comments; to Eric Pierce for help in creating the knock-in mouse; to Cheryl Craft, John Nickerson, and Jack Saari for providing antibodies; to Paul Fitzgerald and Brad Shibata for assistance with electron microscopy; and to Bob Birge for assistance with spectral analysis.

${ }^{*}$ C.I. and L.L.D. contributed equally to this work.

Correspondence should be addressed to: Edward N. Pugh Jr., Departments of Physiology and Cell Biology, University of California, Davis, 1544 Newton Court, Davis, CA 95618. E-mail: enpugh@ucdavis.edu.

DOI:10.1523/JNEUROSCI.0131-12.2012

Copyright $\odot 2012$ the authors $\quad 0270-6474 / 12 / 328094-11 \$ 15.00 / 0$ cones are essential for daytime vision, including color and high acuity foveal vision, cone-specific disease has an especially high impact on visual function.

Many neurodegenerative diseases like retinal degeneration result from an overload of the ER-associated degradation (ERAD) pathway (Lin et al., 2007; Kosmaoglou et al., 2008; Matus et al., 2011). Quality control of nascently translated proteins in the ER is an essential function of all cells (Ellgaard and Helenius, 2003), and ERAD helps ensure that proteins exported from the ER are correctly folded (Ron and Walter, 2007; Vembar and Brodsky, 2008). When ERAD is insufficient to cope with improperly folded proteins, they can collect in the ER, aggregate, and severely disrupt normal cellular function. Because a substantial fraction of proteins are misfolded during biosynthesis (Petaja-Repo et al., 2000; Schubert et al., 2000; Jung et al., 2009), photoreceptors must have an unusually robust ERAD capacity to accompany the high rate of opsin biosynthesis.

We created a knock-in mouse with a point mutation (F81Y) in S-opsin predicted to alter 11-cis-retinal interaction with S-opsin apo-protein and shift the wavelength of maximum sensitivity $\left(\lambda_{\max }\right)$ from the wild-type (WT) value of 360 to $425 \mathrm{~nm}$ (Fasick et al., 2002). Initial characterization revealed that while cones expressing the mutant opsin were morphologically normal and responded to light with the predicted shift in $\lambda_{\max }$, the quantity of F81Y S-opsin expressed was reduced below $50 \%$ of the WT level, with a corresponding reduction in light sensitivity. We thus undertook a series of studies to determine the mechanisms underlying the reduction in F81Y S-opsin expression. 


\section{Materials and Methods}

Generation and discrimination of Opn $1 \mathrm{sw}{ }^{\text {F81Y/F81Y }}$ mice

We created mice with the F81Y mutation in S-opsin because previous heterologous expression in COS cells had shown this mutation to shift the spectral maximum from $360 \mathrm{~nm}$ (ultraviolet) to $\sim 420 \mathrm{~nm}$ (Fasick et al., 2002), close to that of human S-opsin. (Our numbering of residues is based the mouse S-opsin sequence, accession \#NP_031564.1, while that used by Fasick et al. [2002] F86Y, was based on the bovine rhodopsin sequence.) The strategy for introducing the F81Y point mutation into mouse Opn1sw is detailed in Daniele et al. (2011). To create Opn1sw $w^{F 81 Y / F 81 Y}$ mice, homozygous-targeted mice with the Neomycin (Neo) resistance cassette inserted between exons 3 and 4 (Opn1sw $w^{\text {Neo/Neo }}$ ) were bred against mice expressing a transgene for FLP1 recombinase expressed under the human ACTB promoter (\#003800; The Jackson Laboratory). Excision of the Neo selection cassette was confirmed from mouse genomic DNA using PCR. The PCR primer pairs (forward: 5' -AGT ATC GAA GCG AGT ACT ACA CCT GG-3'; reverse: 5' -GCCTTT TGT GTC GTA GCA GACTCT TG$3^{\prime}$ ) detected $O p n 1 s w^{F 81 Y}$ and $O p n 1 s w^{W T}$. The primer pair (forward: same as previous; reverse: $5^{\prime}$-CCC GGT AGA ATT CGA GAA GTT CCT AT-3') detected targeted Opn1sw $w^{N e o}$ and Opn1sw ${ }^{F 81 Y}$. Together these PCR primers were sufficient for discriminating Opn1sw ${ }^{W T}, O p n 1 s w^{N e o}$, and $O p n 1 s w^{F 81 Y}$ alleles. Further crosses of mice with confirmed excision of the Neo selection cassette $\left(O p n 1 s w^{F 81 Y / W T}\right)$ allowed for selection of FLP transgene-negative Opn1sw $w^{F 81 Y / W T}$ mice. The FLP transgene was detected by PCR with primers recommended by The Jackson Laboratory (forward 5' -CAC TGA TAT TGT AAG TAG TTT GC-3'; reverse 5' -CTA GTG CGA AGT AGT GAT CAG G-3').

\section{Quantitation of Opn1sw transcript levels with real-time PCR}

Total RNA was extracted from whole mouse eyes in Eppendorf tubes with plastic homogenizers using the Tri Reagent (Molecular Research Center). After DNAase treatment (DNA-free kit; Applied Biosystems), cDNA was generated from oligo-dT primed total RNA ( $5 \mu \mathrm{g}$ in $15 \mu \mathrm{l}$ for each reaction; SuperScript First-Strand Synthesis System for reverse transcriptase (RT)-PCR; Invitrogen). Taq-Man quantitative PCR (qPCR) was performed on serially diluted samples of cDNA from Opn1sw ${ }^{\text {F81Y/F81Y }}$ and WT littermate eyes with a TaqMan 7500 qRT-PCR System (Applied Biosystems) using exon-spanning probes for mouse visual opsins and $\beta$-actin, with exon boundary and catalog number as follows: rhodopsin (1-2) Mm00520345-m1, M-opsin (1-2) Mm01193546-m1, S-opsin (1-2) Mm01135619_g1, S-opsin (4-5) Mm00432058_m1, $\beta$-actin Mm00607939-s1. Sample dilutions were matched across the two genotypes such that the cDNA used for the qRT-PCR came from equal quantities of total RNA, and we report the cDNA samples in terms of the quantity of ocular RNA from which they were derived. The 96-well plates used for the qRT-PCR reactions were loaded so that each dilution was replicated approximately inversely proportional to the equivalent RNA quantity to increase the reliability of estimates of cycle threshold $(C T)$ for target genes in samples with greater dilution.

CT data were analyzed with the method of Pfaffl (2001), which takes into consideration the efficiency of amplification for the primers. Efficiency $(E)$ is defined as the negative antilog of the slope of a plot of $C T$ versus $\log _{B}$ (sample dilution), i.e., $E=B^{-1 / \text { slope }}$. Thus, if sample dilution is expressed in base 2 units $(B=2)$, a slope of -1 corresponds to perfect efficiency, $E=2$, i.e., a twofold decrease in $C T$ for each twofold increase in cDNA loaded. Suboptimum primer efficiency (in the range of cDNA used) gives rise to slopes $>-1$. With these definitions, the estimate of the ratio of expression of a target versus a reference gene is given by the following:

$$
\text { Expression ratio }=\frac{\left(E_{\text {target }}\right)^{\Delta C T_{\text {target }}}}{\left(E_{\text {ref }}\right)^{\Delta C T_{\text {ref }}}}
$$

where $\Delta C T_{\text {target }}$ is the difference in $C T$ between WT and $O p n 1 s w^{F 81 Y / F 81 Y}$ littermate samples for the target gene (S-opsin) for a given dilution, and $\Delta C T_{\text {ref }}$ is the CT difference for the reference gene ("ref") between the littermate samples. The expression ratio of S-opsin in WT versus F81Y mice was calculated with Equation 1 for each of the cDNA dilutions and averaged over the dilutions to obtain an overall estimate of the expression ratio.

\section{Antibodies}

A number of different primary antibodies were employed in this investigation for immunoblotting, immunoprecipitation (IP), immuno-EM, and immunohistochemistry (IHC). These include three immunopurified polyclonal antibodies raised against mouse S-opsin peptides: against a CT-terminal peptide (rAb-SopsCT; amino acids 317-333, CRKPMADESDVSGSQKT; Yenzym Antibodies; Daniele et al., 2011); against N-terminal peptides—in rabbit (rAb-SopsNT, MSGEDDFYLFQ; Zhu et al., 2003) and in goat (gAb-SopsNT; sc-14363, Santa-Cruz Biotechnology). Additional antibodies used were rabbit anti-M-opsin (rAb-Mops; MAb65696-100, Abcam), rabbit anti-GNAT2 (sc-390; Santa Cruz Biotechnology), mouse antirhodopsin (4D2; Hicks and Molday, [1986]), rabbit anti- $\beta$-actin (Ab 34737; Abcam), mouse anti-VCP (Ab 11433; Abcam), rabbit anti-EDEM1 (E8159; Sigma-Aldrich), rabbit anti-PDE6 $\alpha^{\prime}$ antibody (gift from Tiansen Li), mouse anti-CRALBP (gift from J. Saari; Nawrot et al., [2004]rsqb]), rabbit antiIRBP (gift from John Nickerson), and mouse anti-KDEL ER retention sequence (Ab12223, lot GR14623-2; Abcam). Alexa 555- and Alexa 568conjugated goat anti-rabbit, and Alexa 488 goat anti-mouse were used to detect primary immunosera in IHC (Invitrogen Life Technologies). Goat anti-rabbit IgG coupled to IR Dye680 (92632221; LI-COR) and donkey antigoat and anti-mouse IgGs coupled to IR Dye800 (LI-COR 92632214 and 92632212) were used as secondary antibodies for immunoblotting.

\section{Western blotting and IP}

Dark-adapted retinas were dissected and retinal lysates prepared as previously described (Daniele et al., 2011). Briefly, isolated retinas were processed in $150 \mu$ l extraction buffer $(20 \mathrm{~mm}$ Bis-Tris propane buffer $\mathrm{pH}$ 7.5, $10 \mathrm{~mm}$ dodecyl- $\beta$-maltoside, and $5 \mathrm{~mm} \mathrm{NH}_{2} \mathrm{OH}$ ) supplemented with complete mini protease inhibitor mixture tablets (Roche), homogenized by sonication for $10 \mathrm{~s}$ and clarified by centrifugation $5 \mathrm{~min}$ at $21,000 \mathrm{~g}$. The rhodopsin concentration of retinal lysates was determined with bleaching difference spectroscopy as previously described (Lyubarsky et al., 2004). Quantification of rhodopsin provides a sensitive means of equating retinal protein mass across genotypes: calibration showed that there is $4.2 \mathrm{pmol}$ rhodopsin for each microgram of total retinal protein, and calculations show that the total quantity of cone opsin in the adult WT mouse retina is $\sim 10 \mathrm{pmol}, 1 / 70$ th that of rhodopsin (Daniele et al., 2011). Lysates were brought to a final concentration of 3 pmol rhodop$\sin / \mu \mathrm{l}$ in Novex Tris-glycine SDS sample buffer $(2 \times$; Invitrogen \#LC2676), and serial dilutions of the stock solution were loaded for quantitative immunoblotting in Novex $4-12 \%$ Tris-glycine precast gels (Invitrogen). The quantity of the retinal lysate (expressed in rhodopsin mass) loaded per lane in gels typically ranged in four steps from 7.5 to 60 pmol. For IP, lysates from animals of different genotypes were normalized for rhodopsin content and brought to a final volume of $200 \mu \mathrm{l}$. IP was performed according to manufacturer's protocol using Dynabeads Protein G co-IP kit (Invitrogen). Samples containing $150 \mu$ l of Dynabeads, $3 \mu \mathrm{g}$ of S-opsin C-term antibody and lysate were incubated for 40 $\min$ at room temperature. The supernatant was then removed and the beads washed three times in washing buffer. Elution of the bound proteins was achieved by adding $20 \mu \mathrm{l}$ of elution buffer and $20 \mu \mathrm{l}$ of $2 \times$ SDS sample buffer with $\beta$-mercaptoethanol. Eluted samples were subjected to SDS-PAGE and immunoblotting. Immunopositive bands were visualized with either enhanced chemiluminescence (ECL; Thermo Fisher Scientific) or a LI-COR Odyssey Infra-red imaging system, and quantified with software provided by the manufacturer and cross-checked with customized MATLAB scripts. (For display purposes, but not analysis, images of entire PVDF membranes were inverted and their contrast reduced.)

IHC

Retinal vibratome sections. Mice were sacrificed and eyes enucleated and immersion fixed in 4\% paraformaldehyde (PFA) in $1 \times$ PBS for 30-40 $\mathrm{min}$ at room temperature. The anterior segment was cut away with fine scissors and the lens removed. After three exchanges of PBS, eyecups were embedded in $4 \%$ agarose gel preheated to $70^{\circ} \mathrm{C}$ (low melting; Invitrogen/ 
Invitrogen Life Technologies, Cat. \#18300-012) and oriented as described so that the sectioning plane bisected the dorsoventral midline (Wagner et al., 2000; Daniele et al., 2011), and $150 \mu \mathrm{m}$ sections were made using a vibrating blade microtome (Leica Microsystems, model VT 1000S). Vibratome sections were incubated with primary immunosera at $4^{\circ} \mathrm{C}$ overnight after $\sim 1 \mathrm{~h}$ incubation at room temperature with normal sera (goat, donkey; Jackson ImmunoResearch). Secondary immunosera were applied for $1.5-2 \mathrm{~h}$ at room temperature after three washes in PBS. All immunosera were diluted in a PBS buffer containing bovine serum albumin (BSA; $0.5 \%$ ) and Triton X-100 $(0.5 \%)$. Slide-mounted sections with dual immunofluorescence labeling were visualized with a Zeiss LSM 510 confocal microscope (Zeiss Microscope Imaging). Images were imported into Huygens Software (Scientific Volume Imaging) and deconvolved with a theoretical point spread function. Deconvolved images were exported as RGB TIFs and adjustments to brightness and contrast made with ImageJ software (Rasband WS, ImageJ, US National Institutes of Health, http://rsb.info.nih.gov/ij/, 1997-2009).

Retinal flatmounts. Mouse eyes were enucleated and prefixed for retinal flatmounting in $4 \%$ PFA in $1 \times$ PBS for $1 \mathrm{~h}$ at room temperature. Cuts were made to mark the orientation (as indicated above) of the retina within the eyecup before the retina was separated from the sclera and fixed in $4 \%$ PFA in $1 \times$ PBS overnight at $4^{\circ} \mathrm{C}$. Retinal flatmounts were labeled with an AlexaFluor 488 conjugate of peanut agglutinin (PNA; Invitrogen/Invitrogen) and anti mouse S-opsin (rAb-SopsCT) diluted in PBS buffer with $0.5 \%$ BSA and $0.1 \%$ Triton $\mathrm{X}-100$. After washing with PBS, retinas were incubated with a goat anti-rabbit Alexa 555 secondary antibody for $1 \mathrm{~h}$ at room temperature. Finally, the isolated retinas were flattened, mounted on a microscope slide with a coverslip, and imaged with a Nikon Eclipse TE2000-U microscope equipped for epifluorescence using a $10 \times$ objective. Cone spatial densities were determined from images with a feature detection algorithm using ImageJ software (Rasband WS, ImageJ, NIH, http://rsb.info.nih.gov/ij/, 1997-2009). The ImageJ output data were further refined with customized MATLAB image analysis software (Daniele et al., 2011).

Quantitative analysis of S-opsin immunofluorescence. For quantitative analysis of S-opsin immunofluorescence, vibratome sections of WT and $O p n 1 s w^{F 81 Y / F 81 Y}$ littermates were made and singly labeled in parallel with S-opsin primary antibodies and with an Alexa Fluor 568-conjugated secondary. $Z$-stacks were collected with a photon-counting, two-photon laser scanning microscope operating in its linear range (Calvert et al., 2007). Image acquisition, laser intensity, and scan parameters were controlled by a custom LabVIEW-based interface (Coleman Technologies). The titanium-sapphire laser (Chameleon Ultra II; Coherent) was tuned to $800 \mathrm{~nm}$ for excitation of the Alexa Fluor 568 fluorochrome. Custom MATLAB (MathWorks) scripts (Calvert et al., 2007; Nikonov et al., 2008) were used for segmenting complete cones or oriented "slabs" from the $3 \mathrm{D} Z$-stacks, and for extraction of voxel intensity values (in photon counts) from the $3 \mathrm{D}$ image stacks.

\section{Electron microscopy}

Mice were euthanized by cardiac perfusion following asphyxiation with $\mathrm{CO}_{2}$. Fixatives consisted of $2.5 \%$ glutaraldehyde, 2\% PFA in 0.1 M Na cacodylate, $\mathrm{pH} 7.4$, for conventional electron microscopy (EM) and $0.5 \%$ glutaraldehyde and $4 \%$ PFA in Sorenson buffer for immuno-EM processing. Eyes were dissected into eyecups, divided into wedges centered at the ventral midline, and subjected to overnight fixation. Further processing for conventional EM was performed as described previously (Daniele et al., 2011). Immuno-EM of mouse retinas was performed as described by (Erickson, 1987) using $\mathrm{rAB}-$ SopsCT. Images of the sections were acquired with a transmission electron microscope (CM 120, Phillips Biotwin Lens; FEI) coupled to a digital camera (Gatan MegaScan, model 794/20, digital camera $[2 \mathrm{~K} \mathrm{X} 2 \mathrm{~K}]$ ) at the Diagnostic and Research Electron Microscopy Laboratory at University of California Davis.

\section{Cone electrical recordings}

Light responses of mouse cone photoreceptors were recorded with published methods (Nikonov et al., 2006). In brief, the cell body region of one to several cells in the most distal portion of the outer nuclear layer of a retinal slice was drawn into a suction pipette, and the membrane current measured with a current-to-voltage converter (a form of loose-patch recording). Rod currents were suppressed with a $500 \mathrm{~nm}$ rod-saturating background, and the responses of cones to brief, calibrated flashes of various wavelengths from 340 to $630 \mathrm{~nm}$ measured. Tissue dissection was performed under IR-illumination. Cauterization (Bovie; Aaron Medical) was used to mark the eyecup so that retina orientation could be maintained. To obtain recordings from ventral cones, wedges of retina $(\sim 1 / 8$ area of retina) centered at the ventral midline $\sim 1.5 \mathrm{~mm}$ from the optic nerve to the edge of the retina were cut out of the eyecup and chopped into small pieces $(\sim 200$ $\mu \mathrm{m}$ on a side) with a fine blade.

\section{Extinction coefficient of F81Y S-opsin}

To measure its extinction coefficient, F81Y S-opsin was expressed heterologously and purified. Duplicate plates were transfected with cDNA for WT and F81Y S-opsin (both with C terminus modified for immunopurification with 1D4 monoclonal antibody) using $15 \mu \mathrm{g}$ DNA per $15 \mathrm{~cm}$ plate using procedures described previously (Babu et al., 2001). Cells were cultured for $55 \mathrm{~h}$ at $37^{\circ} \mathrm{C}$ in media containing $10 \%$ newborn calf serum. Culture was done in incubators kept mostly in the dark, but with occasional brief light exposure when the incubator door was opened. Cells were collected and washed in PBS + glucose at $4^{\circ} \mathrm{C}$ extensively. Under dim red light (Kodak \#2 filter), $5 \mu \mathrm{M}$ 11-cis-retinal was then added to the cells followed by overnight incubation at $4{ }^{\circ} \mathrm{C}$; this procedure effectively stops translation while allowing the 11-cis-retinal to combine with competent opsin to form bleachable visual pigment. Cells were collected, washed with PBS + glucose at $4^{\circ} \mathrm{C}$, and then solubilized with $1 \%$ dodecyl maltoside. The cone opsin was purified using a 1D4 Sepharose column, with elution buffer containing $0.1 \%$ dodecyl maltoside at $4^{\circ} \mathrm{C}$. The extinction coefficient of mouse F81Y S-opsin was measured with bleaching by acid denaturation (Babu et al., 2001), and found to be within a few percent of that of WT mouse S-opsin, $\varepsilon_{\max }=41,760$ (Vought et al., 1999; data not shown).

\section{Intraperitoneal injections of 11-cis-retinal}

Littermate pairs of WT and Opn1sw ${ }^{\text {F81Y/F81Y }}$ mice were injected every other day for a $10 \mathrm{~d}$ period with $20 \mu \mathrm{g} 11$-cis-retinal in $100 \mu \mathrm{l}$ vehicle, drawn from a $\sim 40 \mathrm{~mm}$ stock solution in $100 \%$ ultrapure $\mathrm{EtOH}$ purged with argon. (The 11-cis-retinal was provided by Dr. Rosalie Crouch under the auspices of the National Eye Institute.) The injection vehicle consisted of $10 \% \mathrm{BSA}$ in $0.9 \%$ sterile $\mathrm{NaCl}$, and the $\mathrm{EtOH}$ with 11 -cis-retinal constituted $<2 \%$ by volume. For each littermate pair of mice injected with 11-cis-retinal, a yoked control littermate pair of WT and Opn1sw ${ }^{\text {FIIY/F81Y }}$ mice was sham-injected with vehicle containing EtOH alone. During the course of injections mice were housed in a vivarium with a $12 \mathrm{~h}$ light/dark cycle with light intensities of 50-100 lux. Mice were dark adapted overnight before sacrifice for quantification of their cone opsins with Western blotting (as described above).

\section{Results}

\section{F81Y point mutation dramatically reduces S-opsin protein expression}

The gene-targeting strategy used to create the F81Y S-opsin knock-in $\left(O p n 1 s w^{F 81 Y / F 81 Y}\right)$ mouse inserted a Neomycin resistance cassette between the third and fourth exons (Fig. 1A). Successful targeting was confirmed with Southern blotting of ES cells and sequencing of genomic DNA (Fig. $1 B$ ). Surprisingly, initial Western blotting suggested a $>2$-fold reduction in the level of expression of the $O p n 1 s w^{F 81 Y}$ allele relative to the WT allele (Fig. 1C). In contrast, there was no reduction in the expression of $\mathrm{M}$-opsin and conespecific G-protein $\left(\mathrm{G}_{\mathrm{t}} \alpha_{\mathrm{c}}\right)$ and phosphodiesterase (PDE6c) were only modestly reduced by 28 and $26 \%(n=2$ littermate pairs), respectively.

To determine if the decrease in expression of the Opn1sw ${ }^{F 81 Y}$ allele arose from altered transcription efficiency, we performed 
A Opn1swgenelocus

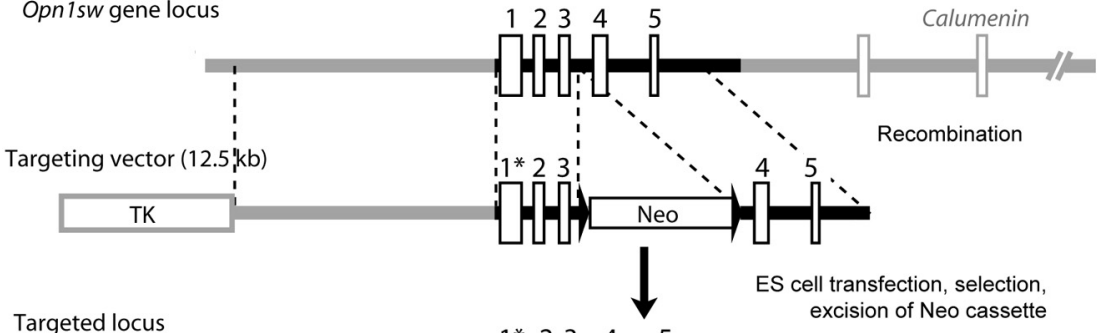

Targeted locus

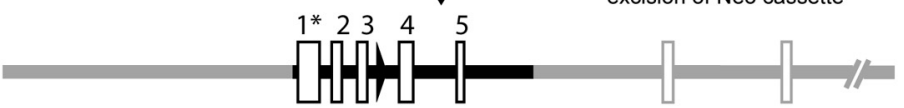

B

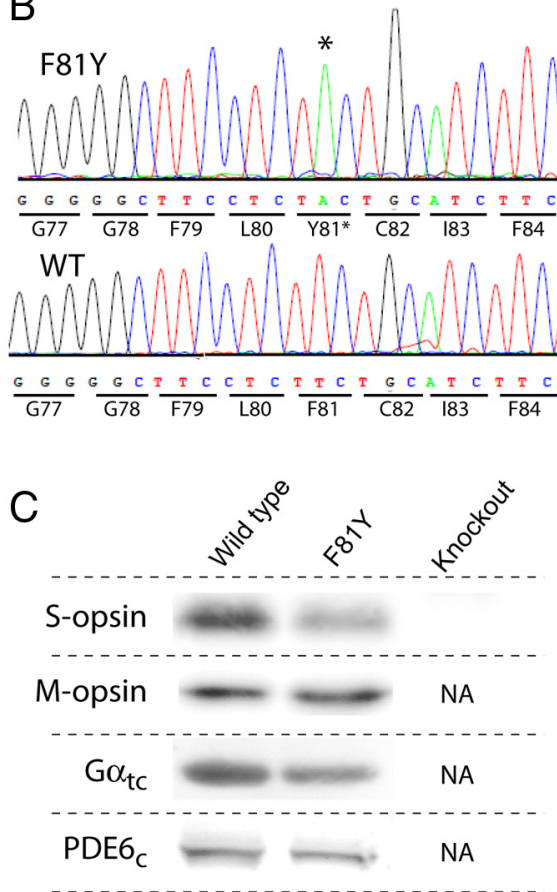

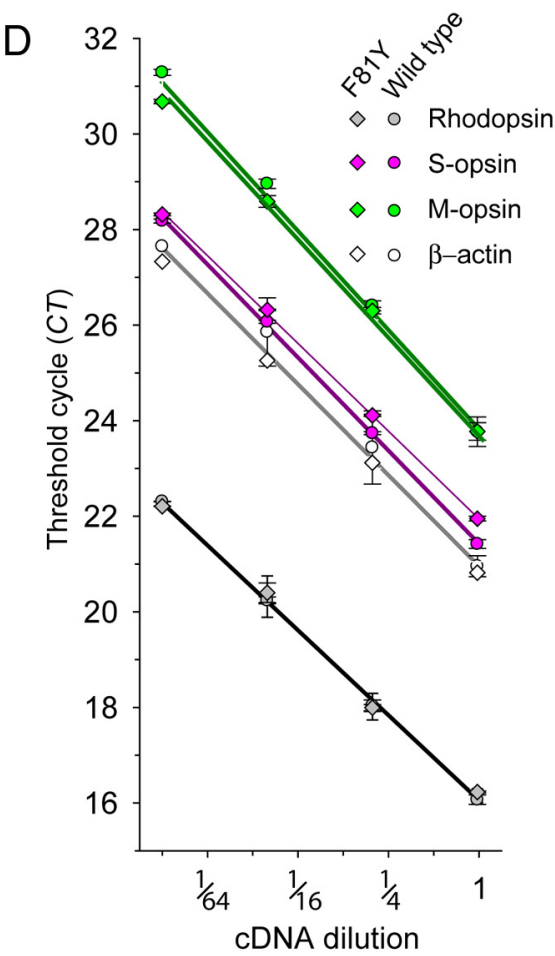

Figure 1. F81Y point mutation in S-opsin leads to reduced expression with negligible mRNA reduction. $\boldsymbol{A}$, Schematic of $0 \mathrm{pn} 1 \mathrm{sw}$ targeting strategy. The targeting vector comprised a Neomycin resistance cassette (Neo) flanked by Flp recombinase target (FRT) recombination sites (arrowheads) and homologous sequences ( $4.5 \mathrm{~kb} 3^{\prime}$ and $816 \mathrm{bp} 5^{\prime}$ ) to the 0pn 7 sw gene locus (the calumenin gene, which abuts the $5^{\prime}$ end of the Opn $1 \mathrm{sw}$ gene is transcribed in the reverse direction on the complementary strand). The asterisk indicates the locus of the targeted point mutation. $\boldsymbol{B}$, DNA sequencing confirmation of the F81Y knockin. Sequences of genomic DNA of an Opn $1 s w^{F 81 Y / F 81 Y}$ and WT littermate pair. $C$, Western blots of S-opsin and other cone-specific proteins. S-opsin blot: lanes of the blots were loaded with $14 \mu \mathrm{g}$ of retinal lysate (60 pmol rhodopsin). The density of the band corresponding to $0 p n 1 s w^{F 81 Y / F 81 Y}$ (middle lane) was reduced $\sim 3$-fold relative to that of the WT littermate control (left lane). Lysate of an S-opsin knock-out mouse retina (right lane) served as a control in this experiment and throughout the work for antibody specificity. M-opsin blot, The same blot was reprobed for M-opsin. Blots comparing $G_{t} \alpha_{c}$ and PDE6 ${ }_{c}$ expression show slight reductions in Opn1sw ${ }^{\text {F81Y/F81Y }}$ retinas relative to WT littermate controls. D, qRT-PCR results. The threshold cycle $(C T)$ of Taq-Man qPCR reactions with primers for rhodopsin (gray-filled symbols), S-opsin (purple filled), M-opsin (green filled), and $\beta$-actin (white filled) message are plotted as a function of the dilution of the reverse-transcribed cDNA. The plotted data are from one experiment with mRNA extracted from a WT and Opn1sw ${ }^{F 81 Y / F 81 Y}$ littermate pair. Error bars indicate SDs for replicates within the experiment. The CT's of F81Y S-opsin are shifted slightly upward relative to the CT's of WT S-opsin. The CT's for rhodopsin are $\sim 6 \log _{2}$ steps lower than those for S-opsin, indicating an $\sim 64$-fold greater abundance for rhodopsin message (given the closely comparable efficiencies of the primers over this range of dilutions).

qRT-PCR analysis of mRNA from retinas of WT and Opn1sw ${ }^{\text {F8IY/F8IY }}$ littermate pairs, using rhodopsin and $\beta$-actin as reference genes (Fig. 1D). Six replications of the experiment with mRNA from the separately analyzed eyes of two littermate pairs yielded an average upward shift in the logarithmic CT of $+0.24 \pm 0.09$ (mean \pm SEM, $p=0.02) \log _{2}$ units for Opn1sw ${ }^{F 81 Y}$ versus WT S-opsin mRNA, corresponding to a $15 \pm 5 \%$ decrease in Opn1sw ${ }^{F 81 Y}$ mRNA. Appli- cation of the method of Pfaffl et al. (2001), which takes into consideration the efficiencies of different PCR primer pairs, yielded the identical estimate of Opn $1 s w^{F 81 Y}$ mRNA reduction, because the efficiencies of amplification of the target and reference genes were within $4 \%$ of ideal (see Materials and Methods). In the same samples, the M-opsin mRNA in Opn1sw ${ }^{F 1 Y / F 81 Y}$ mice was negligibly different from WT $(6 \pm 6 \%)$, and there was no detectable change in rhodopsin mRNA.

To determine precisely the reduction in expression of the protein product of the $O p n 1 s w^{F 81 Y}$ gene we performed quantitative comparisons of S-opsin immunoblots of Opn1sw $w^{F 81 Y / F 81 Y}$ and WT littermate pairs. In each experiment four levels of retinal lysate from an Opn1sw ${ }^{F 81 Y / F 81 Y}$ and WT pair were blotted with anti-S-opsin antibodies, and the expression ratio estimated as the ratio of the slopes of the blot strength versus protein load data for the two genotypes (see Materials and Methods; see Fig. 6). Thirty-four such experiments from 12 littermate pairs revealed the average quantity of S-opsin in Opn1sw F $^{\text {FIY }}$ /F81Y retinas to be reduced $2.6 \pm 0.3$-fold that of WT controls (mean \pm SEM; $p<$ 0.001 , for $t$ test comparison against an expression ratio of unity). In summary, there was a far greater reduction in the Opn1sw ${ }^{F 81 Y}$ protein than in its mRNA, suggesting post-transcriptional downregulation of the mutant gene product.

\section{Cones of Opn1sw ${ }^{F 81 Y / F 81 Y}$ mice have shifted $\lambda_{\max }$ and reduced light sensitivity}

Mouse rods that underexpress rhodopsin have altered outer segment structure and signaling (Calvert et al., 2001; Liang et al., 2004), raising the question of whether the large reduction in the expression of F81Y S-opsin alters the anatomical structure and physiological signaling of Opn1sw $w^{F 81 Y / F 81 Y}$ cones. To assess the functionality of F81Y cone cells, we recorded flash responses from single cones using the suction electrode method (Nikonov et al., 2006). Light responses of cones of the ventral retina of Opn1sw F81Y/F81Y $^{\text {mice were very similar }}$ to those of WT mice (Fig. $2 A, C$, Table 1 ), indicating that the outer segments of Opn1sw ${ }^{\text {F81Y/F81Y }}$ cones are fully functional. As expected (Fasick et al., 2002), the spectral sensitivity of the Opn1sw ${ }^{\text {FIIY/F81Y }}$ cones was red-shifted $60 \mathrm{~nm}$, having a $\lambda_{\max }$ of $420 \mathrm{~nm}$ as compared with the WT value of $360 \mathrm{~nm}$. However, the absolute sensitivity of $O p n 1 s w^{F 81 Y / F 81 Y}$ cones at $\lambda_{\max }$ was $\sim 3$-fold lower than that of WT cones $\left(p<10^{-5}\right.$; Fig. $2 E$, Table 1$)$, consistent with the 2.6-fold reduction in F81Y S-opsin protein expression. 
A
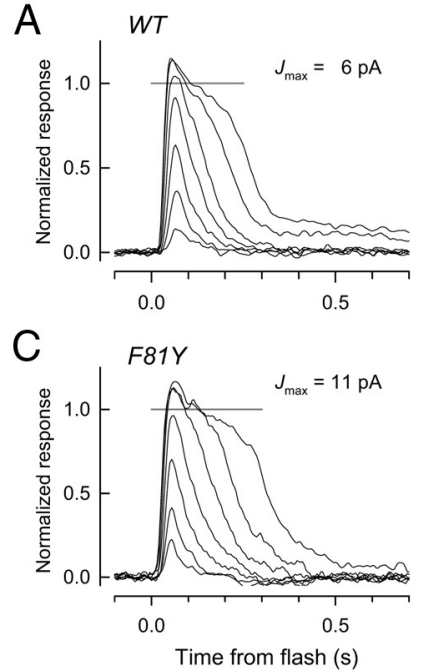

B

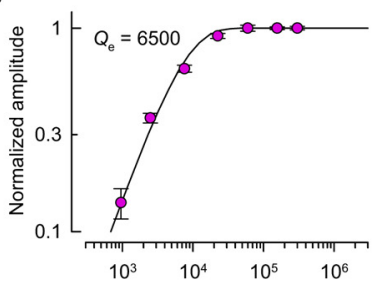

D

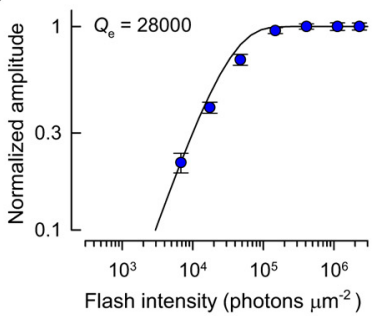

E

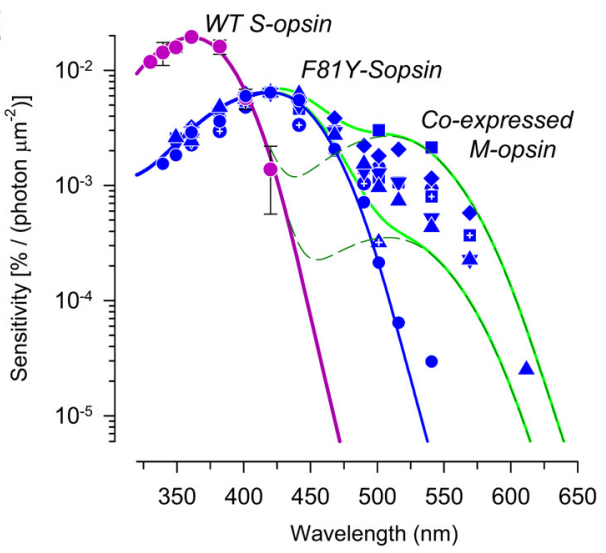

Figure 2. The maximal sensitivity of $O p n 15 W^{F 81 Y / F 81 Y}$ cones is shifted to $420 \mathrm{~nm}$ but reduced threefold. $A, C$, Normalized photocurrents recorded in response to $20 \mu$ s flashes of $360 \mathrm{~nm}(\boldsymbol{A})$ or $420 \mathrm{~nm}(C)$ light of a WT and an Opn $15 W^{F 81 Y / F 81 Y}$ ventral cone (the saturating photocurrent response levels, marked in gray, were 6 and $11 \mathrm{pA}$, respectively). $\boldsymbol{B}, \boldsymbol{D}$, Response versus intensity functions were derived from the corresponding data of $\boldsymbol{A}$ and $\boldsymbol{C}$ and plotted and fitted with exponential saturation functions of the form $r / r_{\max }=1-\exp \left(-Q / Q_{e}\right)$, where $Q_{e}$ is the flash energy density that yields a response of normalized amplitude of $1-\exp (-1)=0.63$. The reciprocal $1 / Q_{e}$ defines flash sensitivity, i.e., the fraction response per unit intensity in the linear range of intensities. $\boldsymbol{E}$, Spectral sensitivities of individual $0 p n 7 s w^{F 81 Y / F 81 Y}$ ventral cones (bluefilled symbols) compared with average data of WT ventral cones. Individual cones were stimulated with light of varied wavelength and graded intensity to extract the flash sensitivity at each wavelength. Sensitivity is plotted on an absolute scale, with WT and Opn $7 \mathrm{sW}^{\mathrm{F} 81 Y / F 81 Y}$ cone data scaled to correspond to the mean sensitivity at the $\lambda_{\max }$ of each population. The colored curves are visual pigment templates (Lamb, 1995) with a $\lambda_{\max }$ of $360 \mathrm{~nm}$ (WT S-opsin, violet), $420 \mathrm{~nm}$ (F81Y S-opsin, blue), and $510 \mathrm{~nm}$ (M-opsin, green). The M-opsin template was added to the $\mathrm{S}$-opsin templates at each of two sensitivities, determined by the upper and lower $99 \%$ confidence intervals of M-opsin coexpression for a large population of WT ventral cones (Daniele et al., 2011). Error bars in $\boldsymbol{B}$ and $\boldsymbol{D}$ indicate SEMs.

Table 1. Properties of ventral cones of Opn 1 sw ${ }^{F 81 Y / F 81 Y}$ mice and WT controls

\begin{tabular}{|c|c|c|c|c|}
\hline Genotype & $N$ & $\begin{array}{l}\text { Maximal current } \\
\text { (pA) }\end{array}$ & $\begin{array}{l}\text { Time to peak of } \\
\text { dim-flash response } \\
\text { (ms) }\end{array}$ & $\begin{array}{l}\text { Sensitivity } \\
{\left[\% /\left(\text { photon } \mu \mathrm{m}^{-2}\right)\right]}\end{array}$ \\
\hline Opn1sw & 8 & $8.5 \pm 1.3$ & $60 \pm 10$ & $(6.1 \pm 0.7) \times 10^{-3}$ \\
\hline WT & 17 & $7.8 \pm 0.6$ & $70 \pm 9$ & $(2.4 \pm 0.02) \times 10^{-2}$ \\
\hline Statistical significance & & n.s. & n.s. & $p<0.0003$ \\
\hline
\end{tabular}

"Dim flashes" are flashes that suppress $20 \%$ or less of the light-sensitive current. Sensitivity was determined from full light response families (Fig. 2A-D), and is specified in terms of the percent of the maximal photocurrent per unit flash intensity for dim flashes. Error terms are SEMs, and statistical tests were two-tailed $t$ tests assuming unequal variance. n.S., not statistically significant; WT, wild type.
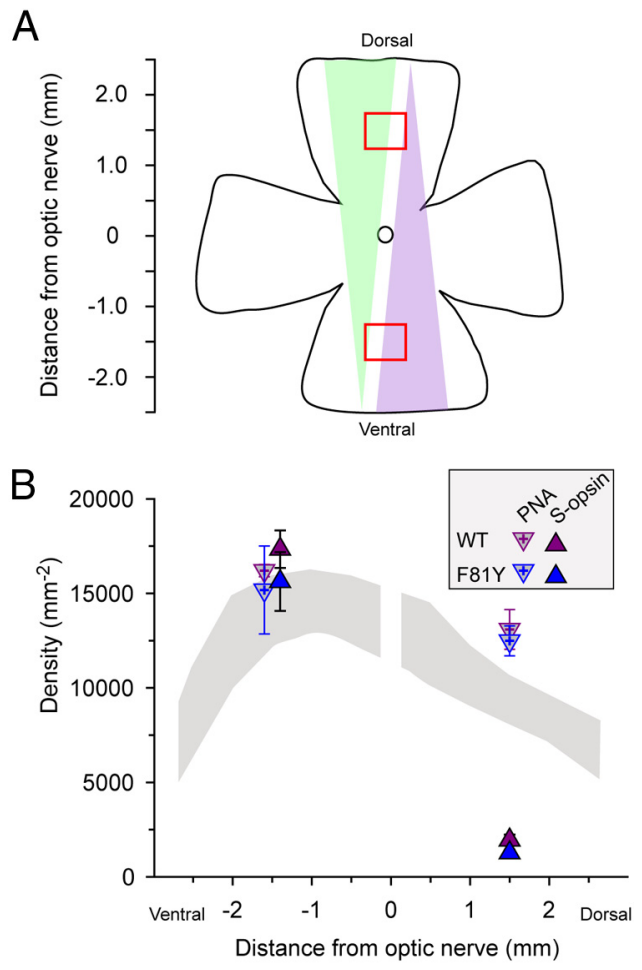

Figure 3. The density of cones expressing $\mathrm{S}$-opsin is normal in $0 p n 7 s w^{F 81 Y / F 81 Y}$ mice. $\boldsymbol{A}$, Schematic of a flatmounted retina oriented with dorsoventral axis running top to bottom. (Precise dorsoventral orientation of the eye was achieved with the method of Wagner et al. [2000]). The two red squares represent the regions centered $1.5 \mathrm{~mm}$ dorsal and $1.5 \mathrm{~mm}$ ventral to the optic disk (central circle) in which cones were counted. Counting was performed in smaller regions at $10 \times$ magnification. $B$, Average cone densities of two littermate pairs (symbols). Error bars indicate SEM. In the ventral retina where virtually all cones strongly express S-opsin, PNA and S-opsin staining are in close agreement for both genotypes. In the dorsal retinal, where M-opsin is predominantly expressed, PNA counts and S-opsin counts diverge. The gray region is an $\sim 90 \%$ confidence interval for cone density of mice of the same genetic background from previous measurements (Daniele et al., 2011). PNA was conjugated with Alexa 488, and S-opsin C-terminal anti-rabbit antibody visualized with an Alexa 555conjugated secondary in a Zeiss LSM-510 microscope.

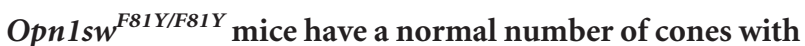 normal length outer segments}

The reduction in S-opsin expression and parallel loss of sensitivity of cones of the Opn1sw ${ }^{F 81 Y / F 81 Y}$ ventral retina reveals that F81Y S-opsin does not traffic to the outer segment in normal quantities, and raises the question of whether the number, size, or other features of the cones are normal in mice expressing the mutant S-opsin. To address this question we analyzed retinal flatmounts and sections immunostained with PNA and S-opsin antibodies. The density of S-opsin-expressing cones in the Opn1sw ${ }^{\text {F81Y/F81Y }}$ retina was normal (Fig. 3). In sections of eyes of littermates selected for adherence of the pigment epithelium, we measured the lengths of Opn1sw ${ }^{F 1 Y / F 81 Y}$ and WT ventral retinal cone outer segments, and found them to be indistinguishable (data not shown): $12.1 \pm 0.02 \mu \mathrm{m}$ (mean \pm SEM, 114 cones, two mice) versus $12.4 \pm 0.02$ (103 cones, three mice), respectively. We conclude that despite the reduction in S-opsin expression, the morphology of ventral cones of Opn1sw $w^{F 81 Y / F 81 Y}$ mice appears normal in light microscopy.

The global reduction in S-opsin expression in Opn1sw ${ }^{\text {F81Y/F81Y }}$ mice measured by Western blotting and reflected in the reduced light sensitivity of cones should also be manifest as a reduction of S-opsin immunofluorescence. To test this prediction, we quantified S-opsin immunolabeled sections of ventral retina of litter- 


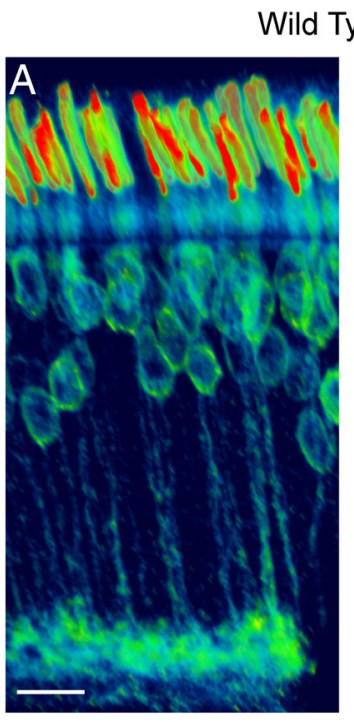

$\mathrm{E}$
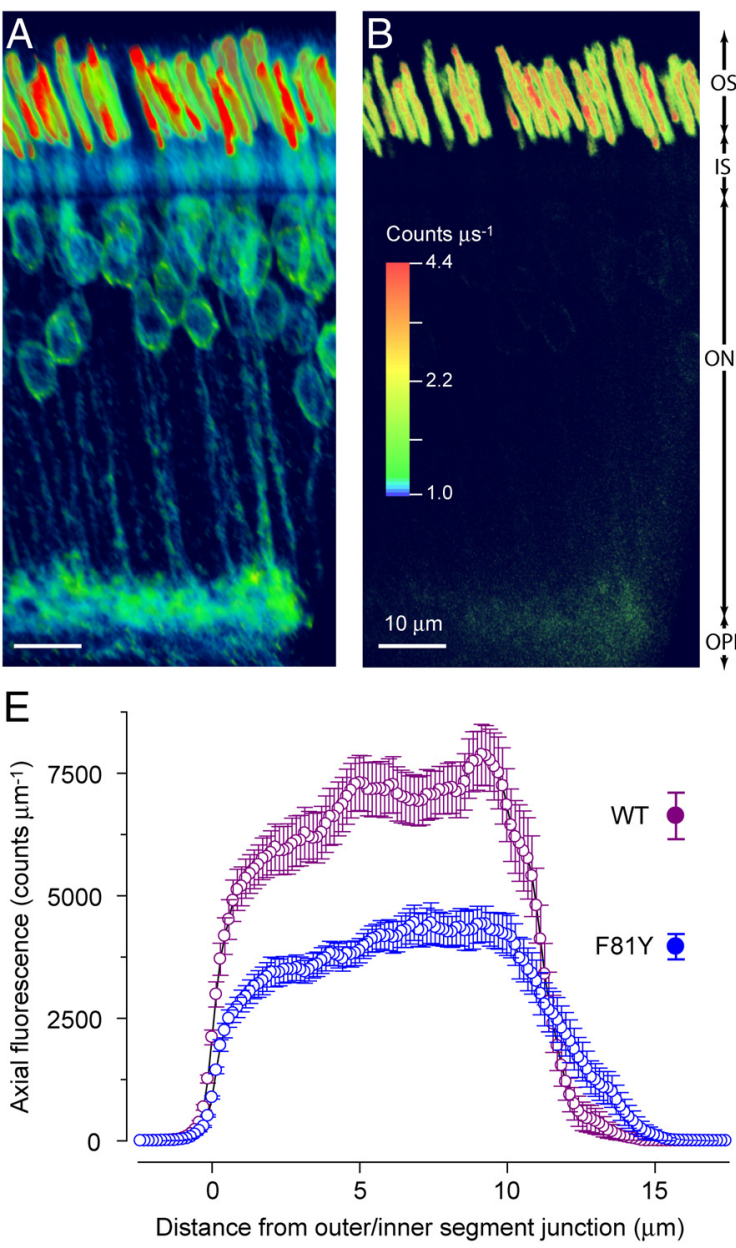

Opn1swF81Y/F81Y
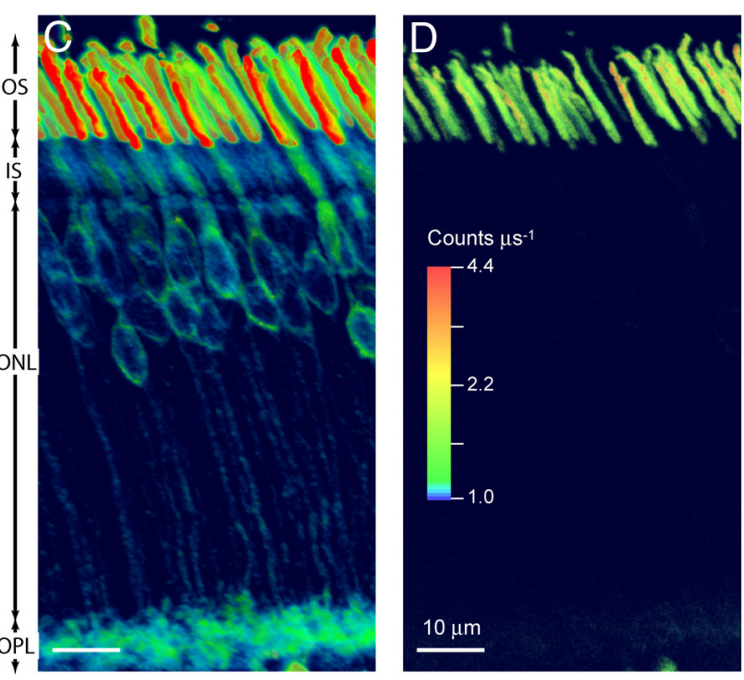

$\mathrm{F}$

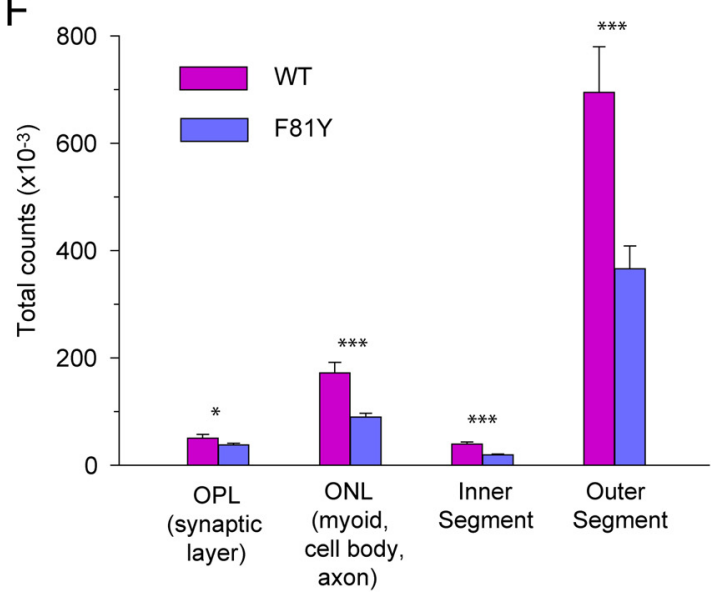

Figure 4. Cones of Opn $1 s w^{F 81 Y / F 81 Y}$ mice have normal length outer segments, with proportionate reduction of S-opsin immunofluorescence throughout the cell. $\boldsymbol{A}-\boldsymbol{D}$, Confocal images of vibratome sections of ventral retina immunoreacted with an N-terminal S-opsin antibody (rAb-SopsinNT) and an Alexa568-conjugated secondary, obtained with a two-photon, laser-scanning photon-counting microscope (see Materials and Methods). The sections were scanned with $800 \mathrm{~nm}$ light at a high $(\boldsymbol{A}, \boldsymbol{C})$ and tenfold lower $(\boldsymbol{B}, \boldsymbol{D})$ laser power, with signal strength coded in pseudocolor. The higher intensity scans $(\boldsymbol{A}, \boldsymbol{C})$ readily reveal the presence of $S$-opsin in all the major compartments but the immunofluorescence of the outer segments saturated the detectors. An absolute scale for the lower intensity scans is provided; calibrations show that the fluorescence at these levels is linearly related to the local concentration of fluorochrome. Scale bar in all panels, 10 $\mu \mathrm{m}$. OS, outer segment; IS, inner segment; ONL, outer nuclear layer; OPL, outer plexiform layer. E, Average axial fluorescence density of WT and Opn1sw ${ }^{F 81 Y / F 81 Y}$ outer segments of the cones ( $n=$ 25 of each genotype) digitally "cut" from the 3D scan matrices of retinal sections from the same littermate pair as illustrated in $\boldsymbol{A}-\boldsymbol{D}$. Error bars indicate SEM. The gradualness of the falloff of fluorescence signal for distances $>10 \mu \mathrm{m}$ arises from the varied length of the outer segments. The spatial averages between 2 and $10 \mu \mathrm{m}$ (filled symbols at right) stand in the ratio $1.6: 1$ (WT:F81Y). $\boldsymbol{F}$, Average absolute fluorescence of subregions of "slabs" (width $\times$ depth $\times$ length $=5 \mu \mathrm{m} \times 5 \mu \mathrm{m} \times \sim 100 \mu \mathrm{m}$ ) of 10 sections of each genotype, scanned as in $\boldsymbol{B}$ and $\boldsymbol{D}$. ${ }^{* * *} p<0.001$; ${ }^{*} p=$ 0.05 .

mate pairs with a photon-counting, two-photon imaging system (Fig. 4). For one littermate pair, the average absolute S-opsin immunofluorescence of Opn1sw ${ }^{F 1 Y / F 81 Y}$ cone outer segments was reduced on average 1.6-fold (Fig. $4 E$; $p<0.00002$ for test of no difference); for a second littermate pair the average reduction was 2.2 fold $(p<0.00003)$.

\section{A substantial fraction of $S$-opsin resides outside the outer segment in both WT and Opn1sw F81Y/F81Y $^{\text {cones }}$}

The apparently normal outer segment morphology of the Opn1sw ${ }^{F 1 Y / F 81 Y}$ cones suggests that opsin and membrane delivery to the outer segment may be to some extent uncoupled in cones. This contrasts with the situation in rods, whose outer segment length, diameter, and disk structure depend on rhodopsin expression level (Liang et al., 2004). This possible difference between rods and cones led us to investigate the subcellular localization of opsin in different compartments of the cones, and in particular in the cell bodies and inner segments, where translation, ER and Golgi sorting, and membrane delivery to the basal disk of the outer segment take place. S-opsin immunofluorescence was readily observed in the cone inner segment, cell body, myoid region, along the axon, and in the synaptic pedicles of both WT and Opn1sw ${ }^{F 81 Y / F 81 Y}$ ventral cones (Fig. $4 A, C$ ). We quantified the axial distribution of S-opsin immunofluorescence in oriented digitally excised slabs of retina: to a first approximation, there was a proportional reduction of S-opsin in each subcellular region of Opn1sw ${ }^{F 81 Y / F 81 Y}$ cones (Fig. $4 F$ ), and in both genotypes in excess of $25 \%$ of the S-opsin immunogenicity was not in the outer segment.

\section{F81Y S-opsin expression is reduced by ERAD}

The decrease in F81Y S-opsin expression, combined with the absence of a comparable decrease in its mRNA (Fig. 1D), suggests that $O p n 1 s w^{F 81 Y}$ mRNA is less efficiently translated 


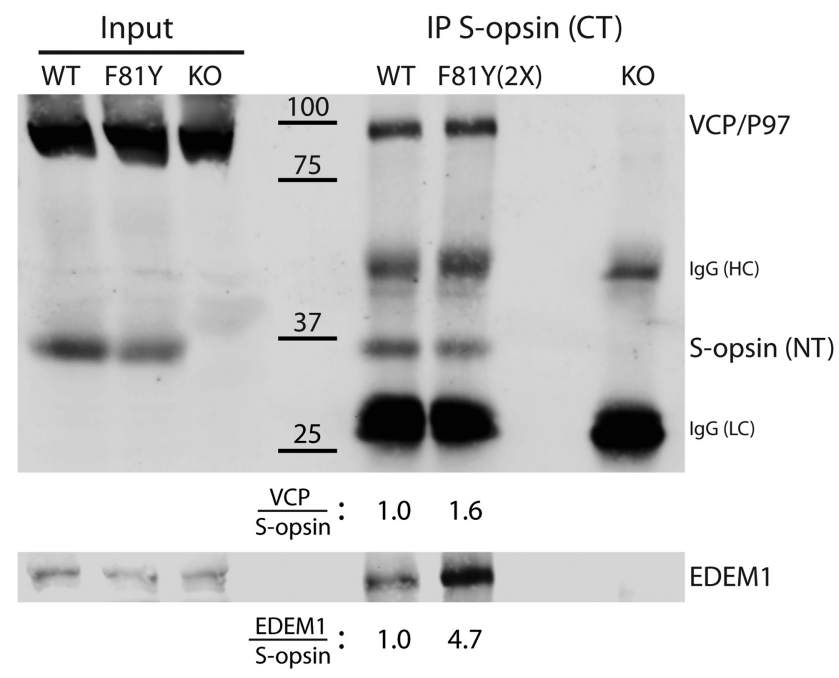

Figure 5. Molecular factors governing ERAD have elevated association with F81Y S-opsin. Results of an experiment in which S-opsin was immunoprecipitated, and the precipitate probed for two markers of ER quality control, EDEM1, and the AAA-ATPase VCP/P97. At left of the size markers are immunoblots for inputs to the assay for WT, Opn1sw ${ }^{\text {FiY/F81Y }}$, and S-opsin-null $(K 0)$, with loads of 21,42 , and $21 \mu \mathrm{g}$ retinal protein, respectively, while to the right are the blots of immunoprecipitates obtained with a C-terminal S-opsin antibody (rAb-SopsCT). (The quantity of the Opn1sw ${ }^{F 81 Y / F 81 Y}$ Iysate was increased $2 \times$ over that for the WT to accommodate the decreased expression level) (Figs. 1, 6). The blots were probed simultaneously with anti-S-opsin ( $g A b-S o p s N T$ ), anti-VCP, and anti-EDEM1 antibodies, with immunofluorescence of the S-opsin and VCP secondaries in the green channel, and the EDEM1 secondary in the red channel, respectively, of the LI-COR system. The ratio of the blot signals for VCP to S-opsin in the immunoprecipitates was measured and found to be enhanced 1.6-fold for F81Y over WTS-opsin, while that for EDEM1 was enhanced 4.7-fold. The ratios of expression of VCP and EDEM1 in the F81Y lysate are given relative to the WT ratio, which is set to unity. (Bands for the lgG heavy and light chains are identified at right).

than the mRNA of WT Opn1sw, or that newly synthesized F81Y S-opsin protein is rapidly degraded after translation. One well characterized pathway that governs protein removal early in the secretory pathway is ERAD (Vembar and Brodsky, 2008). ER degradation enhancing $\alpha$-mannosidase-like 1 (EDEM1) is a lectin chaperone that accelerates ERAD by selectively targeting misfolded proteins to the site of retrotranslocation (Hosokawa et al., 2001; Oda et al. 2003), and has been shown to play a role in targeting immaturely glycosylated P23H rod opsin for ERAD (Kosmaoglou et al., 2009). Another important component of the ERAD retrotranslocation machinery is the transitional AAA-ATPase VCP/P97, which provides the energy required to extract polyubiquitinated substrates through the ER membrane (Ye et al., 2001, 2005), and has been shown to play a role in the degradation of heterologously expressed P23H rod opsin (Griciuc et al., 2010).

To test the hypothesis that the ERAD pathway is involved in the reduction of F81Y S-opsin expression, we performed IP assays of WT and Opn1sw ${ }^{F 81 Y / F 81 Y}$ retinas with an S-opsin antibody to determine whether EDEM1 and VCP are differentially complexed with S-opsin (Fig. 5). VCP and EDEM1 were readily detected in the immunoprecipitates from both WT and Opn1sw ${ }^{F 81 Y / F 81 Y}$ retinas, but were absent from those of S-opsin knock-out mice, establishing the S-opsin specificity of the antibody. Comparing the blot densities of VCP and EDEM1 relative to that of S-opsin in the immunoprecipitates, we found VCP to be enhanced $3.7 \pm 0.7$-fold $(n=5 ; p<0.01)$, while EDEM1 was enhanced $2.6 \pm 0.7$-fold $(n=4 ; p=0.05)$. These results implicate ERAD in the reduced expression of F81Y

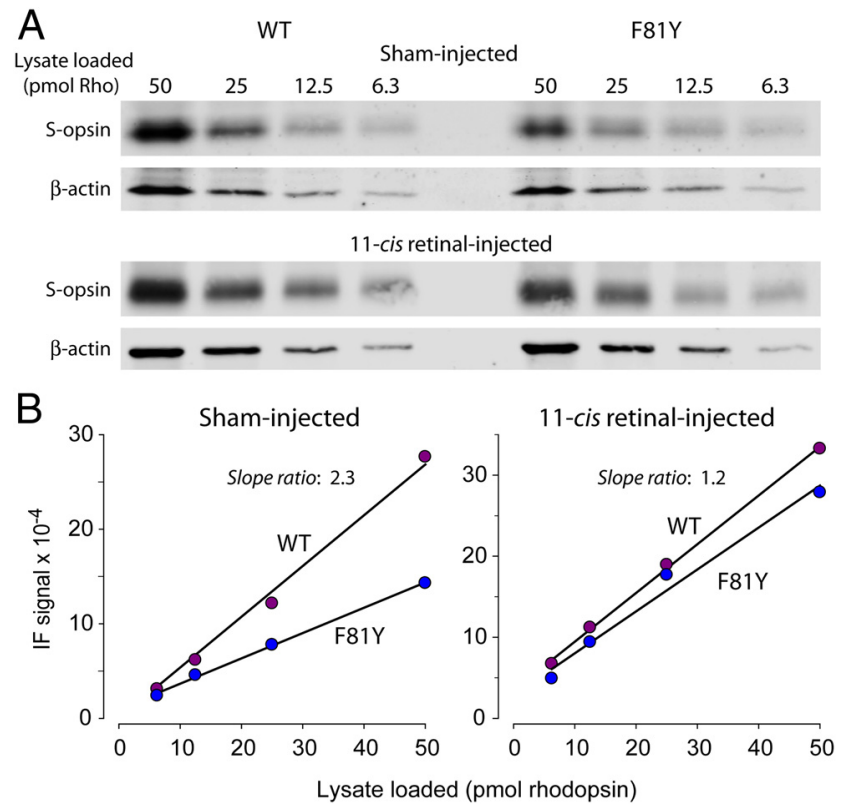

Figure 6. Exogenously increased 11-cis-retinal restores S-opsin expression in Opn $15 W^{\text {F81Y/F87Y }}$ to the WT level. A, Quantification of S-opsin in littermate pairs sham-injected or injected with $20 \mu \mathrm{g}$ 11-cis-retinal in $100 \mu$ l vehicle 5 times on alternative days. Serially diluted retinal lysates of WT and Opn $75 W^{F 87 Y / F 81 Y}$ littermates were analyzed for their S-opsin content in the green channel of the LlCOR imager; $\beta$-actin was measured in the same membranes with the red channel as a secondary loading control for any effect of 11-cis-retinal on rhodopsin. $\boldsymbol{B}$, Plots of the S-opsin blot signals in $\boldsymbol{A}$ with linear regression to extract the slope ratios. The slope ratio for WT versus F81Y S-opsin for the sham-injected mice was 2.3-fold, but the slope ratio approached unity for the 11-cis-retinal-injected mice, indicating that the injections restored the expression of S-opsin in $0 p n 15 w^{887 Y / F 87 Y}$ to a near normal level.

S-opsin, and as a consequence suggest that newly translated F81Y S-opsin has a stronger tendency to misfold than WT S-opsin.

\section{Exogenous 11-cis-retinal, the cone opsin GPCR ligand, increases F81Y S-opsin expression to the WT level}

Because the F81Y point mutation clearly alters the binding pocket for 11-cis-retinal ligand (thus affecting spectral sensitivity), we considered the hypothesis that interaction of the nascently translated S-opsin with 11-cis-retinal might affect the folding of F81Y S-opsin. We tested this hypothesis by systemically increasing 11cis-retinal with intraperitoneal injections in $O p n 1 s w^{F 81 Y / F 81 Y}$ and WT mice. Littermate pairs were injected with $20 \mu \mathrm{g}(70 \mathrm{nmol})$ of 11-cis-retinal on alternate days for $10 \mathrm{~d}$, a time sufficient for complete renewal of their outer segments (Young, 1967, 1971; Hollyfield, 1979; Jonnal et al., 2010); sham-injected, age-matched littermate pairs exposed to the identical light-rearing conditions served as controls. Quantitative analysis of Western blots for S-opsin (Fig. 6) from 10 sets of littermate pairs revealed the ratio of expression of S-opsin in Opn1sw ${ }^{\text {F81Y/F81Y }}$ versus WT for 11-cisretinal-injected mice to be $0.96 \pm 0.06$ (mean \pm SEM), while for the sham-injected mice the corresponding ratio was 0.52 , corresponding to a $1.9 \pm 0.05$-fold reduction in F81Y S-opsin expression, as expected from previous results. The difference between the $\mathrm{S}$-opsin expression ratios of sham-injected and 11-cis-retinalinjected littermate pairs was highly significant $(p<0.001, t$ test for unit ratio). Thus, exogenously elevated 11-cis-retinal caused the expression of F81Y S-opsin to increase to the WT level, effectively rescuing the Opn1sw ${ }^{\text {FS1Y/FS1Y }}$ phenotype of reduced S-opsin expression. 

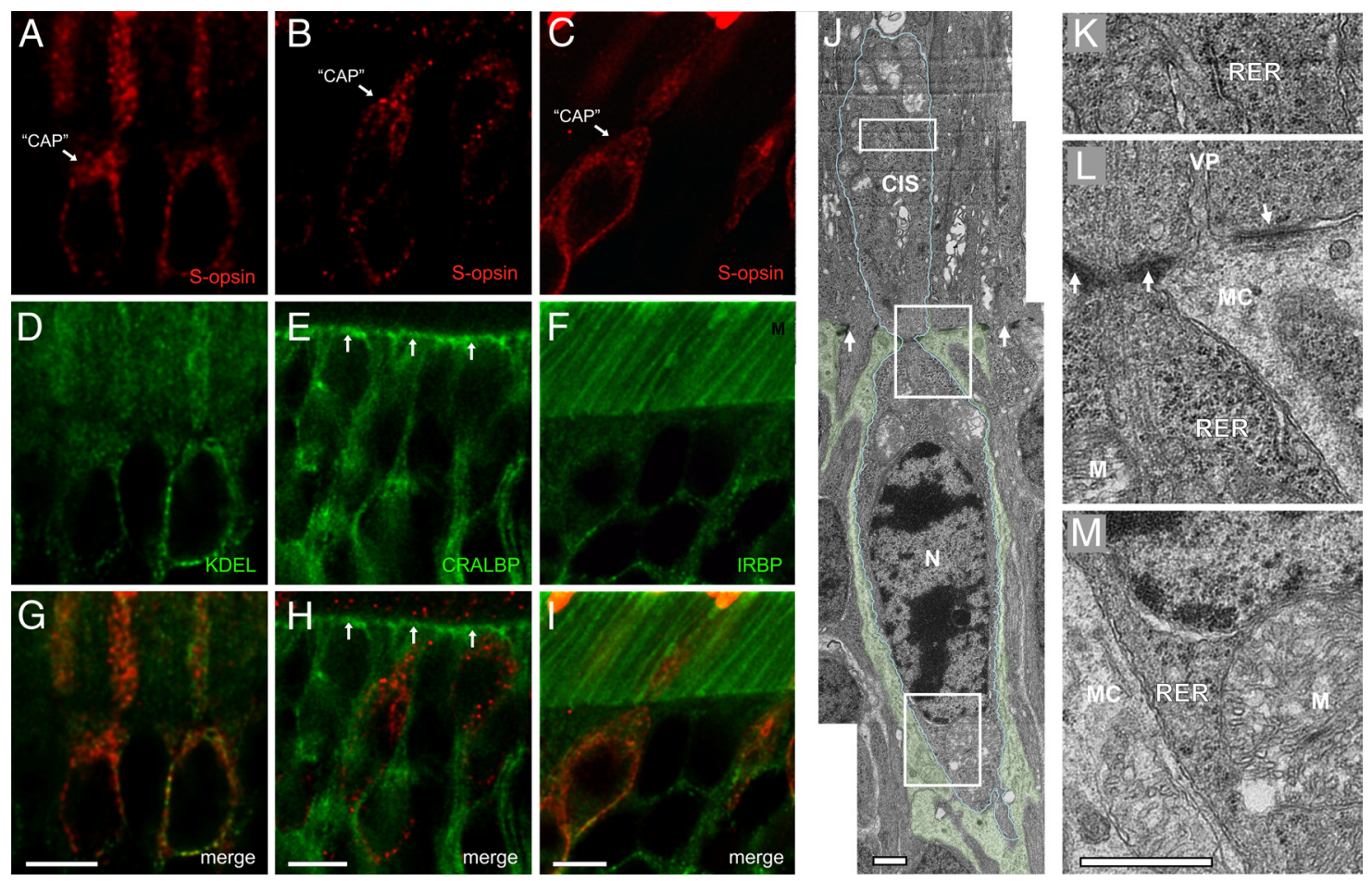

Figure 7. Cellular and subcellular organization of retinal mechanisms implicated in cone opsin biosynthesis. $A-I$, Mouse retinal vibratome sections showing immunohistochemical $S$-opsin localization in the cell body and inner segment, and colocalization with ER ( $\boldsymbol{D}$, anti-KDEL mAb), and with 11-cis-retinoid binding proteins CRALBP (E), and IRBP $(\boldsymbol{F})$. $\mathbf{G}-\boldsymbol{I}$, Merged images of $\boldsymbol{A}$ and $\boldsymbol{D}$, $\boldsymbol{B}$ and $\boldsymbol{E}$, and $\boldsymbol{C}$ and $\boldsymbol{F}$, respectively. The images were taken at $63 \times$ with a Zeiss $L S M-510$ confocal microscope, and were deconvolved with Huygens Professional 3.0 software using the published point spread function. $J$, Electron microscope image of a cone cell body and inner segment (outlined in cyan) and adjoining Mueller cell. The Mueller cell, which has a paler cytoplasm than the cone, has been colorized pale green. $\boldsymbol{A}-\boldsymbol{I} \boldsymbol{I}, \mathrm{Scale}$ bar, $5 \boldsymbol{\mu m}$. $\boldsymbol{K}-\boldsymbol{M}$, Magnified images of the three outlined regions (white rectangles) in $\boldsymbol{J}$ illustrating the high density of rough $E R$. The arrows in $\boldsymbol{E}$ and $\boldsymbol{H}, \boldsymbol{J}$, and $L$ point to adherens junctions, which comprise part of the outer limiting membrane. $K-M$, Scale bar, $1 \mu \mathrm{m}$. CIS, cone inner segment; MC, Mueller cell; M, mitochondrion; $N$, nucleus; RER, rough ER; VP, villous process of Mueller cell.

\section{Exogenous 11-cis-retinal increases the expression of S-opsin in WT mice}

During the course of the 11-cis-retinal injection experiments just reported, we also performed immunoblot comparisons of the quantity of S-opsin extracted from WT mice injected with 11-cisretinal versus WT mice that were sham-injected. These comparisons suggested that the chromophore injections might be increasing the level of WT S-opsin, and so we pursued this effect in a series of experiments with age-matched WT mice. In 16 immunoblot comparisons (Fig. 6) of retinal lysates of nine pairs of WT mice, the ratio of S-opsin in 11-cis-retinal-injected versus sham-injected was $1.22 \pm 0.09$ (mean \pm SEM; $p=0.023$ for one-tailed $t$ test). Thus exogenous 11-cis-retinal increased WT $\mathrm{S}$-opsin expression, and so we conclude that during biosynthesis WT S-opsin, as well as the mutant F81Y-S-opsin, is sensitive to the ambient level of chromophore.

We also compared the quantities of rhodopsin and $\mathrm{M}$-opsin of mice injected with 11-cis-retinal versus mice that were sham-injected. The average quantity of rhodopsin extracted was $430 \pm 25 \mathrm{pmol} /$ eye and $429 \pm 18 \mathrm{pmol} /$ eye (mean \pm SEM, $n=24$ pairs) for sham-injected and 11-cisretinal-injected mice, respectively. The average slope ratio of M-opsin signals in Western blots (Fig. 6) for sham- versus 11-cis-retinal-injected mice was $1.00 \pm 0.06$ (mean \pm SEM, $n=5$ pairs). In both cases, the statistical power was adequate to have reliably detected a $15 \%$ change at $p=0.05$. Thus, increased opsin production with exogenous 11-cis-retinal injections in WT eyes appears uniquely associated with S-opsin. 11-cis-retinoid production by the neural retina may contribute to cone opsin biosynthesis

To more deeply understand the organization of the retinal mechanisms that govern cone opsin biosynthesis and their relationship to native sources of 11-cis-chromophore, we examined the cell body and inner segment regions of cones with high-resolution confocal IHC, and with EM (Fig. 7), taking advantage of the ability of S-opsin antibodies to localize S-opsin in these subcellular regions (Fig. 4). The cell body region contains substantial S-opsin and a high density of rough ER (Fig. 7D, J-M), and every cone cell body had a "cap" region, where rough ER and nonouter segment S-opsin were localized (Fig. 7A-C). Remarkably, Mueller cell processes tightly apposed the ER-containing region of the cones, as revealed by IHC staining for the cellular 11-cis-retinoid binding protein, CRALBP (Fig. 7 B,E,H) (Bunt-Milam and Saari, 1983; Nawrot et al., 2004), and well known morphological features of Mueller cells, including villous processes that project into the inner segment layer (Fig. 7J,L). Mueller cells in some species have been established to be capable of synthesizing 11-cis-retinol (Das et al., 1992), and CRALBP serves as a high-affinity cellular carrier for both 11-cis-retinol and 11-cis-retinal (Futterman et al., 1977; Saari et al., 1982, 2001). In addition, interphotoreceptor retinoid binding protein (IRBP) is clearly present near the cell body and inner segment region of cones (Fig. 7F). Because IRPB is secreted by photoreceptors into the interstitial space, some IRBP is no doubt also present in the cone cytoplasm (Fig. $7 F$ ). Overall, then, these results show that the ER-containing regions of the cone, where its opsin is translated and proofread, are tightly apposed to a source of 11-cis-retinol, an immediate 
A

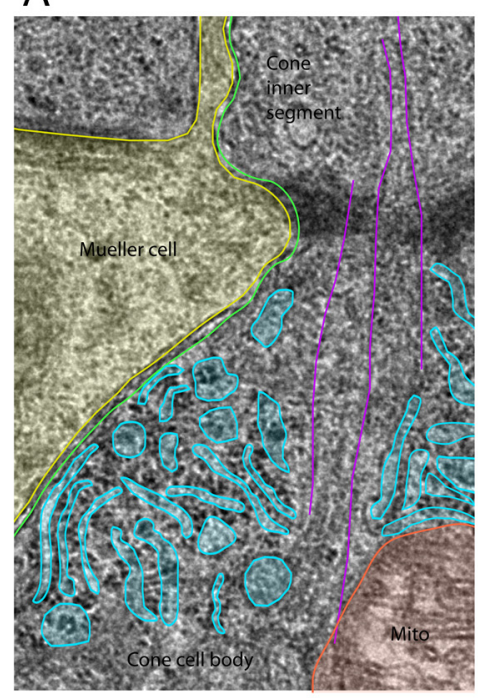

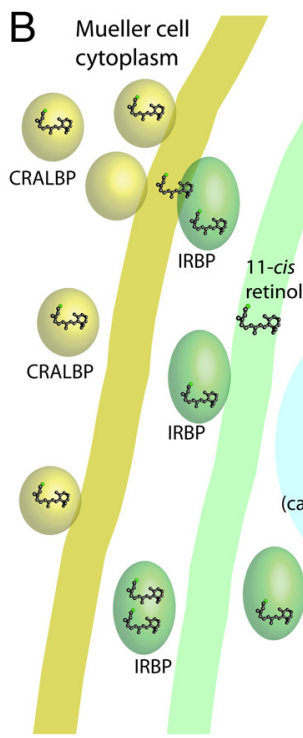

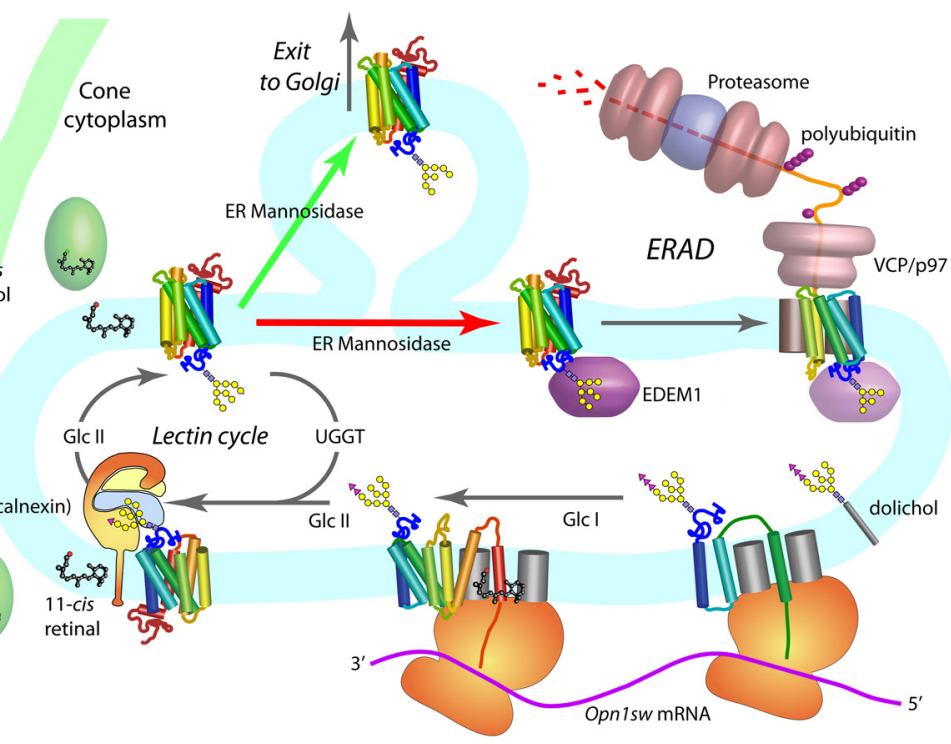

Figure 8. Molecular mechanisms involved in ER quality control of cone opsin biosynthesis. $A$, EM image from Figure 8 L, (reflected) colorized to illustrate key structures. Idealized portions of RER membrane (cyan), drawn to highlight polyribosomes (dark gray densities). The plasma membrane of the Mueller cell is traced in yellow and that of the cone in green. The outer membrane of a mitochondrion is traced in orange, and apparent particle tracks going from the cell body RER to the inner segment are traced in violet (Fig. 8 L). $\boldsymbol{B}$, Molecular mechanisms of ER quality control during cone S-opsin biosynthesis. Translation is initiated as polyribosomes with opsin mRNA dock on the translocon on the ER membrane (lower right). Cotranslationally (lower right), a universal 14-sugar glycosyl tree (glucose, violet triangles; mannose, yellow circles; $\mathrm{N}$-acetylglucosamine, gray squares) is transferred to the $\mathrm{N}$-terminal glycosylation site (N12-I13-S14) from a dolichol intermediate. During translation or shortly thereafter glucosidases (Glc I II) trim 2 of the 3 glucoses. Released from the translocon, the opsin enters into the lectin-binding proofreading cycle in which the final glucose is trimmed or a glucose is transferred (UGGT). The lectin calnexin is illustrated, along with disulfide isomerases (blue, yellow) that it recruits and coordinates. When the final glucose is trimmed by GIc Il and dependent on its folding status, the nascent opsin is reglucosylated (by UGGT) and re-enters the lectin cycle or is prepared for exit from the ER (green arrow), or binds to EDEM1 in preparation for retrotranslocation by VCP, ubiquitination, and proteolysis by the proteasome. Because 11-cis-retinal alters the branching ratio between the ER exit and ERAD pathways, it must interact with the opsin earlier than the branch point, cotranslationally when the Lys 291 on the seventh helix (red) is translated or during the lectin cycle. (The S-opsin structural diagram is based on an S-opsin homology model of rhodopsin.).

precursor of 11-cis-retinal, and that 11-cis-retinoid-specific carriers are present in the Mueller cell cytoplasm and in the nearby interstitial spaces.

\section{Discussion}

Reduced Opn1sw ${ }^{F 81 Y}$ expression is due to ER quality control and altered 11-cis-retinal binding

The results reported here show that in the absence of a normal interaction of nascent S-opsin with 11-cis-retinal, ER quality control mechanisms reduce the quantity of the protein synthesized. Specific support for the hypothesis that F81Y S-opsin expression is reduced by ERAD is provided by the increased IP by F81Y S-opsin of EDEM1 and of VCP/P97 (Figs. 5, 8B). Overall production of F81Y S-opsin is augmented by 11-cis-retinal (Fig. 6), whose precursor is synthesized by the adjacent Mueller cell (Figs. 7, 8A) (Wang and Kefalov, 2009, 2011).

Our results imply that interaction of 11-cis-retinal with nascent opsin can contribute to ER quality control by altering the branching ratio between Golgi exit and ERAD (Fig. $8 B$ ). The first moment in the life of a nascent S-opsin when 11-cisretinal could interact with its normal binding site is when Lys291 on the seventh transmembrane helix is exposed to the ER membrane during exit from the translocon. More likely, 11-cis-retinal binds to the opsin during the lectin cycle: such binding would occur precisely during the critical phase of proofreading following the completion of translation, when the folding occurs that creates the chromophore binding pocket. In the absence of exogenous 11-cis-retinal F81Y S-opsin appears more likely to fold improperly and undergo excess mannose trimming (red arrow), removing the opsin from the lectin cycle, binding EDEM1 (Kosmaoglou et al.,
Table 2. Branching ratio for ER exit versus ERAD

\begin{tabular}{lll}
\hline Genotype and treatment & Total S-opsin (\% re WT) & Branching ratio (ER exit:ERAD) \\
\hline WT + 11-cis-retinal & 122 & $(99: 1)$ \\
F81Y + 11-cis-retinal & 117 & $96: 1$ \\
WT & 100 & $80: 20$ \\
F81Y & 42 & $34: 66$ \\
\hline
\end{tabular}

Entries in column two are mean values determined from Western blot analyses (Fig. 6) of the S-opsin content of mice of the specified genotype and treatment. Assuming that the branching ratio for 11-cis-retinal-treated WT mice is saturated at 99:1, the other ratios follow. Note that the experiments of Figure 6 compare WT and F81Y mice that were both injected with 11-cis-retinal, but other results show that 11-cis-retinal increases the level of WT S-opsin (See Fig. 8 for explanation of the branching ratio.) ERAD, endoplasmic reticulum-associated degradation; WT, wild type.

2009), leading to its progression to retrotranslocation assisted by $\mathrm{VCP} / \mathrm{P} 97$, ubiquitination, and destruction by the proteasome. Since exogenous 11-cis-retinal increases the expression level of F81Y S-opsin to that of correspondingly injected WT mice (Fig. 6), the chromophore effectively biases the branching ratio of the key reactions in ER quality control (Fig. $8 B$, red/green arrows). In these terms, a useful summary of our experiments can be made in terms of the effects of S-opsin genotype (WT vs F81Y S-opsin) and 11-cis-retinal level on the branching ratio (Table 2).

\section{The $O p n 1 s w^{F 81 Y / F 81 Y}$ mouse as a stationary model of mild chromophore deficiency}

Normal production of 11-cis-retinal is required for the health and survival of rod and cone photoreceptors, as well as their signaling of light capture. Cones of mice with genetic deletions of Rpe65 or Lrat-enzymes expressed in the retinal pigment epithelium cells essential for normal production of 11-cis-retinal from retinoid precursors (Jin et al., 2005; Moiseyev et al., 2005; Redmond et al., 
2005) - undergo rapid degeneration, with rod degeneration progressing more slowly (Rohrer et al., 2005; Znoiko et al., 2005; Fan et al., 2008; Zhang et al., 2008). Cone outer segment structure and function in young Rpe65 $5^{-1-}$ and $\mathrm{Lrat}^{-1-}$ mice can to some extent be restored by exogenous delivery of 11-cis-retinal (Rohrer et al., 2005; Zhang et al., 2008). The cone degeneration that occurs in the absence of normal 11-cis-retinal production was initially proposed to be the result of mistrafficking of cone opsins (Rohrer et al., 2005; Zhang et al., 2008). The results presented here are consistent with more recent studies that suggest that 11-cisretinal plays a role in cone opsin synthesis and maturation, likely acting in the ER (Sato et al., 2010; Zhang et al., 2011). Zhang et al. (2011) have hypothesized that S-opsin is distinctly susceptible to forming aggregates during biosynthesis in the absence of chromophore, leading to activation of ER stress pathways, and ultimately resulting in cone photoreceptor degeneration. Remarkably, Opn1sw ${ }^{F 81 Y / F 81 Y}$ cones show no obvious signs of ER stress, or of S-opsin aggregation, and do not degenerate, and apparently have the capacity to eliminate by ERAD a substantial fraction (at least 50\% WT levels) of improperly folded opsin. This suggests that, in the complete absence of 11-cis-retinal synthesis in Rpe $65^{-1-}$ and $\mathrm{Lrat}^{-1-}$ mice, the fraction of opsin that misfolds may be so large that it strains or exceeds the cell's ERAD capacity (Fig. $8 \mathrm{~B}$, red branch).

\section{A possible novel role for the neural retina retinoid cycle: facilitation of opsin biosynthesis}

The neural retina contains a biochemical pathway that can supply 11-cis-retinal for the regeneration of cone pigment that has been bleached by light (Wang and Kefalov, 2009, 2011; Wang et al., 2009). Central to this pathway are the Mueller cells, which generate 11-cis-retinol from retinoid precursors (Das et al., 1992), and strongly express the 11-cis-retinoid-specific binding protein CRALBP (Bunt-Milam and Saari, 1983; Nawrot et al., 2004), which aids in the delivery of 11-cis-retinoid to the cones. Genetic deletion of CRALBP slows both rhodopsin and cone pigment regeneration (Saari et al., 2001). IRBP, which is secreted by rods and cones, has also been shown to be necessary for normal regeneration of cone pigments (Jin et al., 2009; Parker et al., 2011). Our results suggest a new and important role for the Mueller cell supply to the photoreceptor inner segments and cell bodies, namely, the delivery of chromophore to nascent opsin during the proofreading cycle (Fig. 8).

\section{Is ER proofreading of GPCRs with lipophilic ligands generally ligand sensitive?}

GPCRs naturally activated by lipophilic ligands provide an even broader example of ligand sensitivity of GPCR biosynthesis (Nakamura et al., 2010). Such ligands include prostaglandins, leukotrienes, platelet-activating factor, lysophosphatidic acid, and endocannabinoids. The GPCR literature provides many examples in which coculture with cell-permeant natural ligands or synthetic homolog ligands increases the heterologous expression level of mutant GPCRs. These include the vasopressin 2 receptor (Morello et al., 2000), opioid receptors (Chen et al., 2006; Leskelä et al., 2007), melanocortin receptor (René et al., 2010), and mutant rhodopsin (Noorwez et al., 2004; Krebs et al., 2010) The authors of several of these studies have hypothesized that the ligands bind in the ER to unstable folding intermediates, stabilizing them and permitting ER exit. A full understanding of the factors governing the critical branching ratio between ER exit and ERAD (Fig. 8) and ERAD capacity in the native tissues and cell types in which GPCRs are expressed is central to therapeutic intervention strategies for hereditary diseases arising from mutant GPCRs (Wiseman et al., 2007).

\section{References}

Babu KR, Dukkipati A, Birge RR, Knox BE (2001) Regulation of phototransduction in short-wavelength cone visual pigments via the retinylidene Schiff base counterion. Biochemistry 40:13760-13766.

Bunt-Milam AH, Saari JC (1983) Immunocytochemical localization of two retinoid-binding proteins in vertebrate retina. J Cell Biol 97:703-712.

Calvert PD, Govardovskii VI, Krasnoperova N, Anderson RE, Lem J, Makino CL (2001) Membrane protein diffusion sets the speed of rod phototransduction. Nature 411:90-94.

Calvert PD, Peet JA, Bragin A, Schiesser WE, Pugh EN Jr (2007) Fluorescence relaxation in $3 \mathrm{D}$ from diffraction-limited sources of PAGFP or sinks of EGFP created by multiphoton photoconversion. J Microsc 225:49-71.

Carroll J, Baraas RC, Wagner-Schuman M, Rha J, Siebe CA, Sloan C, Tait DM, Thompson S, Morgan JI, Neitz J, Williams DR, Foster DH, Neitz M (2009) Cone photoreceptor mosaic disruption associated with Cys203Arg mutation in the M-cone opsin. Proc Natl Acad Sci U S A 106:20948-20953.

Chen Y, Chen C, Wang Y, Liu-Chen LY (2006) Ligands regulate cell surface level of the human kappa opioid receptor by activation-induced downregulation and pharmacological chaperone-mediated enhancement: differential effects of nonpeptide and peptide agonists. J Pharmacol Exp Ther 319:765-775.

Daniele LL, Insinna C, Chance R, Wang J, Nikonov SS, Pugh EN Jr (2011) A mouse M-opsin monochromat: retinal cone photoreceptors have increased M-opsin expression when S-opsin is knocked out. Vision Res 51:447-458.

Das SR, Bhardwaj N, Kjeldbye H, Gouras P (1992) Muller cells of chicken retina synthesize 11-cis-retinol. Biochem J 285 (Pt 3):907-913.

Ellgaard L, Helenius A (2003) Quality control in the endoplasmic reticulum. Nat Rev Mol Cell Biol 4:181-191.

Erickson PA, Anderson, DH, Fisher SK (1987) Use of uranyl acetate en bloc to improve tissue preservation and labeling for post-embedding immunoelectron microscopy. J Electron Microsc Tech 5:303-314.

Fan J, Rohrer B, Frederick JM, Baehr W, Crouch RK (2008) Rpe65-/- and Lrat-/- mice: comparable models of leber congenital amaurosis. Invest Ophthalmol Vis Sci 49:2384-2389.

Fasick JI, Applebury ML, Oprian DD (2002) Spectral tuning in the mammalian short-wavelength sensitive cone pigments. Biochemistry 41:6860-6865.

Futterman S, Saari JC, Blair S (1977) Occurrence of a binding protein for 11-cis-retinal in retina. J Biol Chem 252:3267-3271.

Gardner JC, Webb TR, Kanuga N, Robson AG, Holder GE, Stockman A, Ripamonti C, Ebenezer ND, Ogun O, Devery S, Wright GA, Maher ER, Cheetham ME, Moore AT, Michaelides M, Hardcastle AJ (2010) $\mathrm{X}$-linked cone dystrophy caused by mutation of the red and green cone opsins. Am J Hum Genet 87:26-39.

Griciuc A, Aron L, Piccoli G, Ueffing M (2010) Clearance of Rhodopsin(P23H) aggregates requires the ERAD effector VCP. Biochim Biophys Acta 1803:424-434.

Hartong DT, Berson EL, Dryja TP (2006) Retinitis pigmentosa. Lancet 368:1795-1809.

Hicks D, Molday RS (1986) Differential immunogold-dextran labeling of bovine and frog rod and cone cells using monoclonal antibodies against bovine rhodopsin. Exp Eye Res 42:55-71.

Hollyfield JG (1979) Membrane addition to photoreceptor outer segments: progressive reduction in the stimulatory effect of light with increased temperature. Invest Ophthalmol Vis Sci 18:977-981.

Hosokawa N, Wada I, Hasegawa K, Yorihuzi T, Tremblay LO, Herscovics A, Nagata K (2001) A novel ER alpha-mannosidase-like protein accelerates ER-associated degradation. EMBO Rep 2:415-422.

Jin M, Li S, Moghrabi WN, Sun H, Travis GH (2005) Rpe65 is the retinoid isomerase in bovine retinal pigment epithelium. Cell 122:449-459.

Jin M, Li S, Nusinowitz S, Lloyd M, Hu J, Radu RA, Bok D, Travis GH (2009) The role of interphotoreceptor retinoid-binding protein on the translocation of visual retinoids and function of cone photoreceptors. J Neurosci 29:1486-1495.

Jonnal RS, Besecker JR, Derby JC, Kocaoglu OP, Cense B, Gao W, Wang Q, 
Miller DT (2010) Imaging outer segment renewal in living human cone photoreceptors. Opt Express 18:5257-5270.

Jung T, Catalgol B, Grune T (2009) The proteasomal system. Mol Aspects Med 30:191-296.

Kosmaoglou M, Schwarz N, Bett JS, Cheetham ME (2008) Molecular chaperones and photoreceptor function. Prog Retin Eye Res 27:434-449.

Kosmaoglou M, Kanuga N, Aguilà M, Garriga P, Cheetham ME (2009) A dual role for EDEM1 in the processing of rod opsin. J Cell Sci 122:4465-4472.

Krebs MP, Holden DC, Joshi P, Clark CL 3rd, Lee AH, Kaushal S (2010) Molecular mechanisms of rhodopsin retinitis pigmentosa and the efficacy of pharmacological rescue. J Mol Biol 395:1063-1078.

Lamb TD (1995) Photoreceptor spectral sensitivities: common shape in the long-wavelength region. Vision Res 35:3083-3091.

Leskelä TT, Markkanen PM, Pietilä EM, Tuusa JT, Petäjä-Repo UE (2007) Opioid receptor pharmacological chaperones act by binding and stabilizing newly synthesized receptors in the endoplasmic reticulum. J Biol Chem 282:23171-23183.

Liang Y, Fotiadis D, Maeda T, Maeda A, Modzelewska A, Filipek S, Saperstein DA, Engel A, Palczewski K (2004) Rhodopsin signaling and organization in heterozygote rhodopsin knockout mice. J Biol Chem 279:48189-48196.

Lin JH, Li H, Yasumura D, Cohen HR, Zhang C, Panning B, Shokat KM, Lavail MM, Walter P (2007) IRE1 signaling affects cell fate during the unfolded protein response. Science 318:944-949.

Lyubarsky AL, Daniele LL, Pugh EN, Jr. (2004) From candelas to photoisomerizations in the mouse eye by rhodopsin bleaching in situ and the light-rearing dependence of the major components of the mouse ERG. Vision Res 44:3235-3251.

Matus S, Glimcher LH, Hetz C (2011) Protein folding stress in neurodegenerative diseases: a glimpse into the ER. Curr Opin Cell Biol 23:239-252.

Moiseyev G, Chen Y, Takahashi Y, Wu BX, Ma JX (2005) RPE65 is the isomerohydrolase in the retinoid visual cycle. Proc Natl Acad Sci U S A 102:12413-12418.

Morello JP, Salahpour A, Laperrière A, Bernier V, Arthus MF, Lonergan M, Petäjä-Repo U, Angers S, Morin D, Bichet DG, Bouvier M (2000) Pharmacological chaperones rescue cell-surface expression and function of misfolded V2 vasopressin receptor mutants. J Clin Invest 105:887-895.

Nakamura M, Yasuda D, Hirota N, Shimizu T (2010) Specific ligands as pharmacological chaperones: The transport of misfolded G-protein coupled receptors to the cell surface. IUBMB Life 62:453-459.

Nawrot M, West K, Huang J, Possin DE, Bretscher A, Crabb JW, Saari JC (2004) Cellular retinaldehyde-binding protein interacts with ERMbinding phosphoprotein 50 in retinal pigment epithelium. Invest Ophthalmol Vis Sci 45:393-401.

Nikonov SS, Kholodenko R, Lem J, Pugh EN Jr (2006) Physiological features of the $\mathrm{S}$ - and $\mathrm{M}$-cone photoreceptors of wild-type mice from singlecell recordings. J Gen Physiol 127:359-374.

Nikonov SS, Brown BM, Davis JA, Zuniga FI, Bragin A, Pugh EN Jr, Craft CM (2008) Mouse cones require an arrestin for normal inactivation of phototransduction. Neuron 59:462-474.

Noorwez SM, Malhotra R, McDowell JH, Smith KA, Krebs MP, Kaushal S (2004) Retinoids assist the cellular folding of the autosomal dominant retinitis pigmentosa opsin mutant $\mathrm{P} 23 \mathrm{H}$. J Biol Chem 279:16278-16284.

Oda Y, Hosokawa N, Wada I, Nagata K (2003) EDEM as an acceptor of terminally misfolded glycoproteins released from calnexin. Science 299: $1394-1397$.

Parker R, Wang JS, Kefalov VJ, Crouch RK (2011) Interphotoreceptor retinoid-binding protein as the physiologically relevant carrier of 11-cisretinol in the cone visual cycle. J Neurosci 31:4714-4719.

Petaja-Repo UE, Hogue M, Laperriere A, Walker P, Bouvier M (2000) Export from the endoplasmic reticulum represents the limiting step in the maturation and cell surface expression of the human delta opioid receptor. J Biol Chem 275:13727-13736.

Pfaffl MW (2001) A new mathematical model for relative quantification in real-time RT-PCR. Nucleic Acids Res 29:e45.

Rakoczy EP, Kiel C, McKeone R, Stricher F, Serrano L (2011) Analysis of disease-linked rhodopsin mutations based on structure, function, and protein stability calculations. J Mol Biol 405:584-606.
Redmond TM, Poliakov E, Yu S, Tsai JY, Lu Z, Gentleman S (2005) Mutation of key residues of RPE65 abolishes its enzymatic role as isomerohydrolase in the visual cycle. Proc Natl Acad Sci U S A 102:13658-13663.

René P, Le Gouill C, Pogozheva ID, Lee G, Mosberg HI, Farooqi IS, Valenzano KJ, Bouvier M (2010) Pharmacological chaperones restore function to MC4R mutants responsible for severe early-onset obesity. J Pharmacol Exp Ther 335:520-532.

Rohrer B, Lohr HR, Humphries P, Redmond TM, Seeliger MW, Crouch RK (2005) Cone opsin mislocalization in Rpe65-/- mice: a defect that can be corrected by 11-cis retinal. Invest Ophthalmol Vis Sci 46:3876-3882.

Ron D, Walter P (2007) Signal integration in the endoplasmic reticulum unfolded protein response. Nat Rev Mol Cell Biol 8:519-529.

Saari JC, Bredberg L, Garwin GG (1982) Identification of the endogenous retinoids associated with three cellular retinoid-binding proteins from bovine retina and retinal pigment epithelium. J Biol Chem 257:13329-13333.

Saari JC, Nawrot M, Kennedy BN, Garwin GG, Hurley JB, Huang J, Possin DE, Crabb JW (2001) Visual cycle impairment in cellular retinaldehyde binding protein (CRALBP) knockout mice results in delayed dark adaptation. Neuron 29:739-748.

Sato K, Nakazawa M, Takeuchi K, Mizukoshi S, Ishiguro S (2010) S-opsin protein is incompletely modified during $\mathrm{N}$-glycan processing in Rpe65(-/-) mice. Exp Eye Res 91:54-62.

Schubert U, Antón LC, Gibbs J, Norbury CC, Yewdell JW, Bennink JR (2000) Rapid degradation of a large fraction of newly synthesized proteins by proteasomes. Nature 404:770-774.

Vembar SS, Brodsky JL (2008) One step at a time: endoplasmic reticulumassociated degradation. Nat Rev Mol Cell Biol 9:944-957.

Vought BW, Dukkipatti A, Max M, Knox BE, Birge RR (1999) Photochemistry of the primary event in short-wavelength visual opsins at low temperature. Biochemistry 38:11287-11297.

Wagner E, McCaffery P, Dräger UC (2000) Retinoic acid in the formation of the dorsoventral retina and its central projections. Dev Biol 222:460 - 470.

Wang JS, Kefalov VJ (2009) An alternative pathway mediates the mouse and human cone visual cycle. Curr Biol 19:1665-1669.

Wang JS, Kefalov VJ (2011) The cone-specific visual cycle. Prog Retin Eye Res 30:115-128.

Wang JS, Estevez ME, Cornwall MC, Kefalov VJ (2009) Intra-retinal visual cycle required for rapid and complete cone dark adaptation. Nat Neurosci 12:295-302.

Wiseman RL, Powers ET, Buxbaum JN, Kelly JW, Balch WE (2007) An adaptable standard for protein export from the endoplasmic reticulum. Cell 131:809-821.

Ye Y, Meyer HH, Rapoport TA (2001) The AAA ATPase Cdc48/p97 and its partners transport proteins from the ER into the cytosol. Nature 414:652656.

Ye YH, Shibata Y, Kikkert M, van Voorden S, Wiertz E, Rapoport TA (2005) Recruitment of the p97 ATPase and ubiquitin ligases to the site of retrotranslocation at the endoplasmic reticulum membrane. Proc Natl Acad Sci U S A 102:14132-14138.

Young RW (1967) The renewal of photoreceptor cell outer segments. J Cell Biol 33:61-72.

Young RW (1971) The renewal of rod and cone outer segments in the rhesus monkey. J Cell Biol 49:303-318.

Zhang H, Fan J, Li S, Karan S, Rohrer B, Palczewski K, Frederick JM, Crouch RK, Baehr W (2008) Trafficking of membrane-associated proteins to cone photoreceptor outer segments requires the chromophore 11-cisretinal. J Neurosci 28:4008-4014.

Zhang T, Zhang N, Baehr W, Fu Y (2011) Cone opsin determines the time course of cone photoreceptor degeneration in Leber congenital amaurosis. Proc Natl Acad Sci U S A 108:8879-8884.

Zhu X, Brown B, Li A, Mears AJ, Swaroop A, Craft CM (2003) GRK1dependent phosphorylation of $S$ and $M$ opsins and their binding to cone arrestin during cone phototransduction in the mouse retina. J Neurosci 23:6152-6160.

Znoiko SL, Rohrer B, Lu K, Lohr HR, Crouch RK, Ma JX (2005) Downregulation of cone-specific gene expression and degeneration of cone photoreceptors in the Rpe65-/- mouse at early ages. Invest Ophthalmol Vis Sci 46:1473-1479. 